\title{
AN OPTIMAL GALERKIN-HOMOTOPY ASYMPTOTIC METHOD APPLIED TO THE NONLINEAR SECOND-ORDER BVPS
}

\author{
JALIL MANAFIAN
}

\begin{abstract}
In this paper, a well-known optimal Galerkin-homotopy asymptotic method (OGHAM) has been used to solve the nonlinear secondorder boundary value problems (BVPs) derived from the problem of thermo-geometric fin parameter together. The obtained solution has been placed by iteration in each equation of the system. The error function of each equation in the supposed interval has been gained in each iteration of this method and has been selected as an optimal iteration of GHAM to solve the mentioned system by considering the minimum error in the first subinterval. Using the selected iteration of OGHAM, a Multi-step homotopy asymptotic method (HAM) has been applied with a few steps. The proposed method is tested upon nonlinear BVPs from the literature and the results are compared with the available approximate solutions including exact analytical method [1] and THAM [56]. The results indicate that the solution error in the proposed technique has decreased significantly.
\end{abstract}

\section{Introduction}

The mathematical models including the problem of forced convection in a porous duct for the case of slug flow(Darcy model) have great use in industries and engineering. Effecting the incorporation of the boundary and inertial on the fully developed momentum transfer equation in porous media along with major changes and adding the viscous shear stress term where has been used by Nield [50] and using the nonlinear term to account for the form drag effects where has been noted by Nield and Bejan [51] is the problem considering both boundary and inertial effects. Vafai and Kim have obtained an exact solution for forced convection in a channel filled with a porous medium [61]. Thermal dispersion effects on fully developed forced convection inside a porous-saturated pipe are investigated in [62]. Also, Abbsasbandy et al. [1] have reported the exact analytical solution of forced convection in a porous-saturated duct. For further convey on the problem of forced convection refer to Refs. ([10, 37, 49, 52, 57]). Fins are an integral part of any equipment which requires the transfer of heat between the

2010 Mathematics Subject Classification. 65D19, 65H10, 35A20, 35A24, 35C08, 35G50.

Key words and phrases. Optimal Galerkin-homotopy asymptotic method, nonlinear secondorder BVPs. 
solid surface and the ambient atmosphere [8]. There has been a lot of research in this in the past several years. Moreover, in experimental analysis, Baby and Balaji [8] studied the performance of finned heat sinks made of Aluminium used in portable electronic devices. Among one can state fins are employed to enhance the heat transfer between the primary surface and its convective, radiating or convective-radiating environment. The regular perturbation method and a numerical method were investigated by Aziz and Hug [7] to compute a closed form solution for a straight convecting fin with temperature-dependent thermal conductivity. The Adomian decomposition method (ADM) has been used to evaluate the efficiency of straight fins with temperature-dependent thermal conductivity and to determine the temperature distribution within the fin [4]. The efficiency of applicable fins with temperature-dependent thermo-physical properties was reported by well-known authors of literature $([9,55,58])$.

There are many semi-analytical and numerical methods for solving nonlinear partial differential equations (PDEs), some of these methods which solve PDEs are: the Adomian decomposition method [59], the homotopy analysis method $[14,15]$, the homotopy perturbation method [12], the variational iteration method [13, 16, 17, 18, 25, 47], the Laplace Adomian decomposition method [42], the optimal homotopy and differential transform methods [53], the semi-analytical iterative technique [60], and so on. Recently, Marinca and Herisanu have introduced the OHAM $[29,30,31,44,45]$ for the solution of nonlinear problems which made the perturbation methods independent of the assumption of small parameters. In order to achieve this goal, various methods have been developed for linear and nonlinear equations such as optimal homotopy asymptotic method. For further information see references therein $([2,3,19,24,35])$. Also, for further investigations on some valuable researches the interested author refer to Refs. ([6]-[43]). The matched asymptotic expansion method was applied for small values of the Darcy number. Also, the Darcy number of the solution for the Brinkman-Forchheimer momentum equation was found in terms of an asymptotic expansion by Hooman [32]. A perturbation based analysis to investigate forced convection in a porous saturated tube was investigated by Hooman and Ranjbar [33]. Also, thermal dispersion effects on fully developed forced convection in a porous-saturated pipe were investigated using numerical and asymptotic techniques in [34]. Magyari and co-authors [41] investigated the forced convection problem for range $\lambda \geq 0$ of the temperature exponent, the analytical results were near with numerical findings and for range $\lambda<-12$, the existence of a new class of unique solutions, while for $-12<\lambda<0$ the occurrence of multiple solutions was reported.

In order to understand these intricate phenomena, it is key to construct more exact solutions of NLPDEs. By using the obtained semi-analytical solutions one can understand the complex structure of physical phenomena. It is notable that many NLPDEs in diverse fields like biology, physics and chemistry consist of unknown functions and parameters and the study of exact solutions provides the guidance to the researchers to maintain and design the experiments, by producing the suitable natural environment, to obtain the these unknown function and parameters. The betterment of mathematical approaches for finding out a general and compact class of numerical or semi-analytical solutions is one of the 
most basic task to observe the whole dynamical process modeling by complicated NLPDEs from the recent few years. Finding the semi-analytical solutions of NLPDEs has the importance to discuss the stability of numerical solutions and also development of a broad range of new scholar to simplify the routine calculation. Therefore, the foremost concern for the researchers is to find the exact solutions of the nonlinear second-order boundary value problems. For this sake different powerful techniques have been developed for detecting the semi-analytic solutions of the nonlinear second-order boundary value problems by using various symbolic computation like Mathematica, Matlab and Maple. Furthermore, the theory of BVPs draw the attention of the researchers and scientific community, because it is an active area of research in the fields of mechanical engineering and mathematical physics. The thermal conductivity of the fin material are significant in one-dimensional energy balance equation due to this feature. The nonlinear wave phenomena can be examined in various scientific fields such as, fluid dynamics, plasma physics, solitary waves, nonlinear equations, etc.

The objective of the present work is to solve the nonlinear second-order boundary value problems using an analytical technique, optimal Galerkin-homotopy asymptotic method. Therefore, the goal of this article is to seek semi-analytical wave solutions which are in the series form solutions. The basic feature of proposed technique is to observe some elementary relationships between NLPDEs and others simple NLODEs. It has been examined that with the aid of simple solutions and solvable ODEs, different kind of traveling wave solutions of some complicated NLPDEs can be easily constructed. The primary benefit of applying this technique is that we have succeeded in a single move, to gather various types of new solutions in the new form of OGHAM. An important aspect of mentioned technique is to provide us a guideline that how to organize these solutions.

The organization of the paper is as follows. In Sect. 2, we describe the basic formulation in terms of mathematical model. In Sect. 3, we introduce the optimal Galerkin-homotopy asymptotic method and show how the method is used to solve the nonlinear boundary-value problem. Section 4 is devoted to the solution of non-linear boundary-value problem. Some numerical examples are presented in Sect. 5. Finally, Sect. 6 provides conclusions of the study.

\section{Mathematical formulation}

2.1. The Darcy-Brinkman-Forchheimer momentum equation. Consider a steady state pressure driven fully-developed parallel flow through a horizontal channel that is filled with porous media [32], as illustrated in Figure 1. One can see in Figure (1) that the lower and upper plates are placed at $y=-h$ and $y=h$ respectively. For $x>0$, the heat flux at the tube wall is held constant at the specified value. The flow is in the direction of $x$-axis and also the velocity is of the form $v=(u(y), 0,0)$. The flow inside the channel is known to be governed by the Darcy-Brinkman-Forchheimer momentum equation [5]

$$
\frac{d^{2} u^{*}}{d y^{* 2}}=-\frac{G}{\mu_{e f f}}+\frac{u^{*}}{M K}+\frac{\rho C_{f}}{\mu_{e f f} \sqrt{K}} u^{* 2},
$$

where $C_{f}$ is the form drag coefficient, $G$ is the pressure gradient, $K$ is the permeability, $\rho$ is the fluid density, $\mu$ is the fluid viscosity, $\mu_{e f f}$ is the viscosity of 
the fluid in the porous medium and $M\left(=\mu_{e f f} / \mu\right)$ is the viscosity ratio. By considering the following transformations

$$
y^{*}=y h, \quad u^{*}=\frac{G h^{2} u}{\mu},
$$

then Eq. (2.1) reduced to

$$
\frac{d^{2} u(y)}{d y^{2}}-s^{2} u(y)-F s u(y)^{2}+\frac{1}{M}=0,
$$

subject to the initial and boundary conditions

$$
u(1)=0, \quad u^{\prime}(0)=0,
$$

where $F$ is the Forchheimer number and $s$ is the porous medium shape parameter. Awartani and Hamdan [5] investigated numerically utilizing a second-order accurate finite difference technique for solving the Darcy-Brinkman-Forchheimer equation. The asymptotic aspects of solutions to Eq. (2.3) were determined by perturbation schemes for limiting cases by Hooman [32]. Motsa et al. [48] have applied the spectral-homotopy analysis method in order to achieve a solution with good accuracy. Abbasbandy [1] obtained a closed form solution of forced convection in a porous-saturated duct.

2.2. Fin temperature distribution. Take the one-dimensional energy balance equation where illustrated in Figure (2), is considered as

$$
A_{c} \frac{d}{d \xi}\left[k(T) \frac{d T}{d \xi}\right]-P h\left(T_{b}-T_{a}\right)=0 .
$$

By assuming be a linear function of the thermal conductivity of the fin material as

$$
k(T)=k_{a}\left[1+\lambda\left(T-T_{a}\right)\right],
$$

where $k_{a}$ is the thermal conductivity at the ambient fluid temperature of the fin and $\lambda$ is the parameter portraying the variation of the thermal conductivity. Also, by employing the dimensionless parameters as below form

$$
\theta=\frac{T-T_{a}}{T_{b}-T_{a}}, \quad x=\frac{\xi}{b}, \quad \beta=\lambda\left(T_{b}-T_{a}\right), \quad \psi=\left(\frac{h P b^{2}}{k_{a} A_{c}}\right)^{\frac{1}{2}},
$$

then, fin efficiency of convective straight fins with temperature-dependent thermal conductivity is considered as [4]

$$
\beta\left(\frac{d \theta(x)}{d x}\right)^{2}+(1+\beta \theta) \frac{d^{2} \theta(x)}{d x^{2}}-\psi^{2}=0,
$$

under the below boundary conditions

$$
\left.\frac{d \theta(x)}{d x}\right|_{x=0}=0, \quad \theta(1)=1,
$$

where $\psi$ is the thermo-geometric fin parameter and $\beta$ is the thermal conductivity parameter. The ADM and the homotopy perturbation technique were used to evaluate the efficiency of straight fins with temperature-dependent thermal conductivity, respectively, in [4] and [54]. The Taylor series expansion method was employed to the fin problem with a temperature-dependent thermal conductivity 


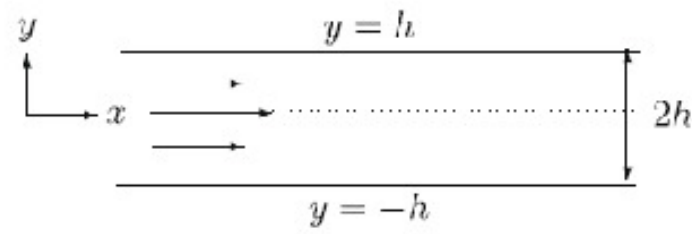

FigURE 1. Steady state parallel flow inside a fluid-saturated porous channel.

has been investigated by Kim and Huang [39]. Analysis convective straight fins with temperature-dependent thermal conductivity were applied by the help of the variational iteration method and the HPM [20] (Ganji et al.), FEM analyzes [11] (Coşkun and Atay) and HAM [36, 38] (Inc and Khani et al.).

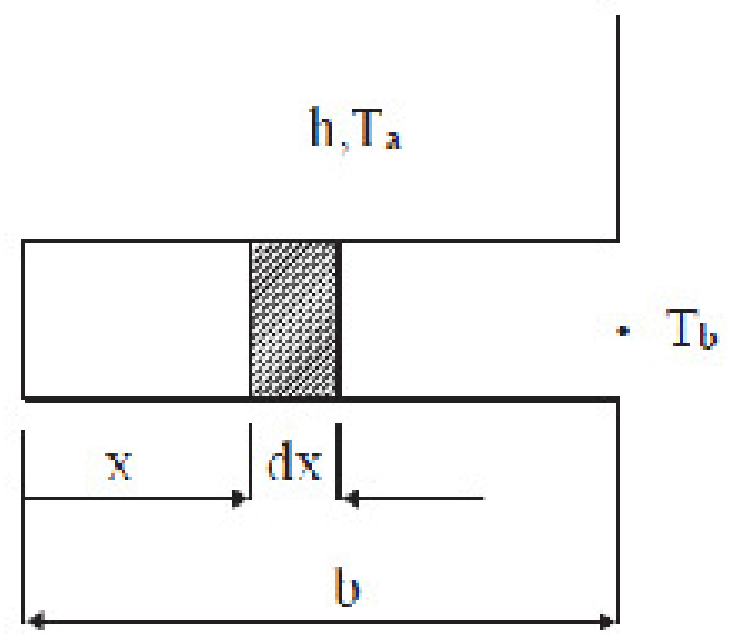

Figure 2. Geometry of a straight fin.

\section{Analysis of the method}

The method of optimal homotopy asymptotic method has been proposed by Marinca and Herisanu [45] in 2008 for the first time and was found on the concept of homotopy. Bring up the partial differential equation of the below form:

$$
\mathcal{K}(v(x))+g(x)+\mathcal{M}(v(x))=0, \quad x \in \Sigma, \quad \mathcal{S}\left(v, \frac{d v}{d x}\right)=0,
$$

where $\mathcal{K}$ is a linear operator and $\mathcal{M}$ is a nonlinear operator. $\mathcal{S}$ is boundary operator, $v(x)$ is an unknown function, and $x$ defines spatial variable, $\Sigma$ is the problem domain and $r(x)$ is a known function.

Based on the OHAM, one can create the optimal homotopy $\rho(x ; q): \Sigma \times[0,1] \rightarrow \mathbb{R}$ which complies:

$$
(1-q)\{\mathcal{K}(\rho(x ; q))+r(x)\}=\Lambda(q)\{\mathcal{K}(\rho(x ; q))+\mathcal{M}(\rho(x ; q))+r(x)\},
$$




$$
\mathcal{S}\left(\rho(x ; q), \frac{\partial \rho(x ; q)}{\partial x}\right)=0,
$$

where $q \in[0,1]$ is an embedding parameter, $\Lambda(q)$ is a nonzero auxiliary function for $q \neq 0, \Lambda(0)=0$. Eq. (3.2) is named the optimal homotopy equation. Vividly, we can write:

$$
\begin{gathered}
q=0 \Rightarrow \mathcal{K}(\rho(x ; 0))+r(x)=0, \\
q=1 \Rightarrow \mathcal{K}(\rho(x ; 1))+\mathcal{M}(\rho(x ; 1))+r(x)=0 .
\end{gathered}
$$

Vividly, until $q=0$ and $q=1$ it holds that $\rho(x ; 0)=\alpha_{0}(x)$ and $\rho(x ; 1)=\alpha(x)$, respectively. Therefore, as $q$ varies from 0 to 1 , the solution $\rho(x ; q)$ comes close from $\alpha_{0}(x)$ to $\alpha(x)$, where $\alpha_{0}(x)$ is achieved from Eq. (3.2) for $q=0$ :

$$
\mathcal{K}\left(\alpha_{0}(x)\right)+r(x)=0, \quad \mathcal{S}\left(\alpha_{0}, \frac{d \alpha_{0}}{d x}\right)=0 .
$$

Next, we pick out the auxiliary function $\Lambda(q)$ in the form $[29,30]$

$$
\Lambda(q)=q A_{1}+q^{2} A_{2}+\ldots+q^{m} A_{m},
$$

where $A_{1}, A_{2}, \ldots, A_{m}$ are constants to be determined. $\Lambda(q)$ can be stated in plenty of the forms as reported by $\mathrm{V}$. Marinca et al. [45, 31, 44].

To access an approximate solution, we enlarge $\rho\left(x ; q, A_{i}\right)$ in Taylor's series about $q$ in the below form as,

$$
\rho\left(x ; q, A_{i}\right)=\alpha_{0}(x)+\sum_{k=1}^{\infty} \alpha_{k}\left(x ; A_{i}\right) q^{k}, \quad i=1,2, \ldots .
$$

Substituting Eq. (3.7) into Eq. (3.2) and equating the coefficient of like powers of $q^{k}$, we catch Zeroth order problem, given by Eq. (3.5), the first and second order problems are given by Eqs. (3.8) and (3.9) respectively and the general governing equations for $\alpha_{k}(x)$ are defined via Eq. (3.10):

$$
\begin{gathered}
\mathcal{K}\left(\alpha_{1}(x)\right)=A_{1} \mathcal{M}_{0}\left(\alpha_{0}(x)\right), \quad \mathcal{S}\left(\alpha_{1}, \frac{d \alpha_{1}}{d x}\right)=0 \\
\mathcal{K}\left(\alpha_{2}(x)\right)-\mathcal{K}\left(\alpha_{1}(x)\right)=A_{2} \mathcal{M}_{0}\left(\alpha_{0}(x)\right)+A_{1}\left[\mathcal{K}\left(\alpha_{1}(x)\right)\right. \\
\left.+\mathcal{M}_{1}\left(\alpha_{0}(x), \alpha_{1}(x)\right)\right], \mathcal{S}\left(\alpha_{2}, \frac{d \alpha_{2}}{d x}\right)=0 \\
\mathcal{K}\left(\alpha_{k}(x)\right)-\mathcal{K}\left(\alpha_{k-1}(x)\right)=A_{k} \mathcal{M}_{0}\left(\alpha_{0}(x)\right)+\sum_{i=0}^{k-1} A_{i}\left[\mathcal{K}\left(\alpha_{k-i}(x)\right)\right. \\
\left.+\mathcal{M}_{k-i}\left(\alpha_{0}(x), \alpha_{1}(x), \ldots, \alpha_{k-i}(x)\right)\right], k=2,3, \ldots \mathcal{S}\left(\alpha_{k}, \frac{d \alpha_{k}}{d x}\right)=0
\end{gathered}
$$

where $\mathcal{M}_{k-i}\left(\alpha_{0}(x), \alpha_{1}(x), \ldots, \alpha_{k-i}(x)\right)$ is the coefficient of $q^{k-i}$ in the expansion $\mathcal{M}(\rho(x ; q))$ about the embedding parameter $q$.

$$
\mathcal{M}\left(\psi\left(x ; q, A_{i}\right)\right)=\mathcal{M}_{0}\left(\alpha_{0}(x)\right)+\sum_{k \geq 1} \mathcal{M}_{k-i}\left(\alpha_{0}(x), \alpha_{1}(x), \ldots, \alpha_{k}(x)\right) q^{k} .
$$


Theorem 3.1. The convergence of the series in Eq. (3.7) depends upon the auxiliary constants $A_{1}, A_{2}, \ldots, A_{m}$ and it is convergent at $q=1$, when we have:

$$
\rho\left(x ; A_{i}\right)=\alpha_{0}(x)+\sum_{k=1}^{\infty} \alpha_{k}\left(x, A_{1}, A_{2}, \ldots, A_{k}\right) .
$$

The consequence of the mth order approximations are specified

$$
\widetilde{\alpha}\left(x, A_{1}, A_{2}, \ldots, A_{m}\right)=\alpha_{0}(x)+\sum_{k=1}^{m} \alpha_{k}\left(x, A_{1}, A_{2}, \ldots, A_{k}\right) .
$$

Substituting Eq. (3.13) into Eq. (3.1), it consequences the residual as below form: $\mathcal{E}\left(x, A_{1}, A_{2}, \ldots, A_{m}\right)=\mathcal{K}\left(\widetilde{\alpha}\left(x, A_{1}, A_{2}, \ldots, A_{m}\right)\right)+r(x)+\mathcal{M}\left(\widetilde{\alpha}\left(x, A_{1}, A_{2}, \ldots, A_{m}\right)\right)$.

Proof. If $\mathcal{E}=0$, then $\widetilde{\alpha}$ will be the closed form of solution. Commonly it does not happen, particularly in nonlinear problems. In order to detect the optimal amounts of $A_{i} ; i=1,2,3, \ldots, m$, we first create the below functional,

$$
\mathcal{F}\left(A_{1}, A_{2}, \ldots, A_{m}\right)=\int_{\Omega} \mathcal{E}^{2}\left(x, A_{1}, A_{2}, \ldots, A_{m}\right) d x,
$$

and then minimizing it, we have

$$
\frac{\partial \mathcal{F}}{\partial A_{1}}=\frac{\partial \mathcal{F}}{\partial A_{2}}=\ldots=\frac{\partial \mathcal{F}}{\partial A_{m}}=0 .
$$

The constants $A_{i}$ can also be detected by another method as below form:

$$
\mathcal{E}\left(\sigma_{1} ; A_{i}\right)=\mathcal{E}\left(\sigma_{2} ; A_{i}\right)=\ldots=\mathcal{E}\left(\sigma_{m} ; A_{i}\right)=0, \quad i=1,2, \ldots, m,
$$

where $\sigma_{i} \in \Sigma$ is in the domain of the problem.

For further information see references therein $([26,27,28])$.

\section{Application of the OG-HAM}

4.1. Application of the OG-HAM for DBF momentum equation. We utilize the offered method (OG-HAM) to solve the DBF momentum equation where given by Eq. (2.3) and (2.4).

Theorem 4.1. Following the OG-HAM formulation, we have:

$$
\mathcal{K}(u(y))=\frac{d^{2} u(y)}{d y^{2}}, \quad \mathcal{M}(u(y))=-s^{2} u(y)-F s u(y)^{2}+\frac{1}{M},
$$

under the below initial condition:

$$
u^{\prime}(0)=0, \quad u(1)=0 .
$$

The second order approximate solution by OHAM for, $q=1$, is;

$$
\widetilde{u}\left(y, A_{1}, A_{2}\right)=u_{0}(y)+u_{1}\left(y, A_{1}\right)+u_{2}\left(y, A_{1}, A_{2}\right) .
$$

With the domain $\Sigma=[a, b]=[0,1]$, the residual will be as

$$
\mathcal{R}\left(y, A_{1}, A_{2}\right)=\frac{d^{2} u\left(y, A_{1}, A_{2}\right)}{d y^{2}}-s^{2} u\left(y, A_{1}, A_{2}\right)-F s u\left(y, A_{1}, A_{2}\right)^{2}+\frac{1}{M} .
$$


Zeroth-order problem defined via Eq. (3.5) is as

$$
\frac{d^{2} u_{0}(y)}{d y^{2}}=0, \quad u_{0}^{\prime}(0)=0, \quad u_{0}(1)=0,
$$

from which we obtain

$$
u_{0}(y)=0 .
$$

First-order problem given by Eq. (3.8):

$$
\begin{gathered}
\frac{d^{2} u_{1}\left(y, A_{1}\right)}{d y^{2}}=\left(1-A_{1}\right) \frac{d^{2} u_{0}(y)}{d y^{2}}+A_{1} s^{2} u_{0}(y)+A_{1} s F\left(u_{0}(y)\right)^{2}-\frac{A_{1}}{M}, \\
u_{1}^{\prime}(0)=0, \quad u_{1}(1)=0 .
\end{gathered}
$$

We access the solution as below:

$$
u_{1}\left(y, A_{1}\right)=-\frac{1}{2} A_{1}\left(y^{2}-1\right) .
$$

Second-order problem given via Eq. (3.9):

$$
\begin{gathered}
\frac{d^{2} u_{2}\left(y, A_{1}, A_{2}\right)}{d y^{2}}=\left(1-A_{1}\right) \frac{d^{2} u_{1}\left(y, A_{1}\right)}{d y^{2}}+A_{1} s u_{1}\left(y, A_{1}\right)\left(2 F u_{0}(y)+s\right)-\frac{A_{2}}{M} \\
+A_{2}\left(s F\left(u_{0}(y)\right)^{2}+s^{2} u_{0}(y)-\frac{d^{2} u_{0}(y)}{d y^{2}}\right), \quad u_{2}^{\prime}(0)=0, \quad u_{2}(1)=0 .
\end{gathered}
$$

The solution becomes:

$$
\begin{gathered}
u_{2}\left(y, A_{1}, A_{2}\right)=-\frac{1}{20 M}\left\{0.0833 A_{1}^{2} s^{2} y^{4}+\left(A_{2}-0.5 A_{1}^{2} s^{2}+A_{1}-A_{1}^{2}\right) y^{2}\right. \\
\left.-A_{2}-A_{1}+A_{1}^{2}+0.4166 s^{2} A_{1}^{2}\right\} .
\end{gathered}
$$

Using Eqs. (4.6), (4.8) and (4.10), the second order approximate solution by OHAM for, $q=1$, is;

$$
\widetilde{u}\left(y, A_{1}, A_{2}\right)=u_{0}(y)+u_{1}\left(y, A_{1}\right)+u_{2}\left(y, A_{1}, A_{2}\right) .
$$

Following the procedure described in Section 3 on the domain $\Sigma=[a, b]=[0,1]$, using the residual,

$$
\mathcal{R}\left(y, A_{1}, A_{2}\right)=\frac{d^{2} u\left(y, A_{1}, A_{2}\right)}{d y^{2}}-s^{2} u\left(y, A_{1}, A_{2}\right)-F s u\left(y, A_{1}, A_{2}\right)^{2}+\frac{1}{M} .
$$

Case A: $F=M=1$

By considering the obtained values of $A_{i}$ 's our approximate solution and Residual for the following subcases, respectively, become,

Subcase I-A: $s=0.0$

$$
A_{1}=1.0, A_{2}=-3.0 \times 10^{-10}, \widetilde{u}(y)=0.4999-0.4999 y^{2}, \widetilde{R}(y)=4.0 \times 10^{-10} \text {. }
$$

Subcase II-A: $s=0.5$

$$
\begin{gathered}
A_{1}=1.33377, \quad A_{2}=0.14232, \quad \widetilde{u}(y)=0.42280-0.40427 y^{2}-0.01853 y^{4}, \\
\widetilde{E}(y)=-0.09301+0.22056 y^{2}-0.14313 y^{4}-0.01498 y^{6}-0.00034 y^{8} .
\end{gathered}
$$


Subcase III-A: $s=1.0$

$$
\begin{gathered}
A_{1}=0.94689, A_{2}=0.02424, \widetilde{u}(y)=0.32392-0.28656 y^{2}-0.03736 y^{4}, \\
\widetilde{E}(y)=-0.00195+0.02389 y^{2}-0.02055 y^{4}-0.02141 y^{6}-0.00139 y^{8} .
\end{gathered}
$$

Subcase IV-A: $s=1.5$

$$
\begin{gathered}
A_{1}=0.73459, A_{2}=0.05293, \widetilde{u}(y)=0.23829-0.18770 y^{2}-0.05059 y^{4}, \\
\widetilde{E}(y)=0.003260 .05056 y^{2}+0.09714 y^{4}-0.02849 y^{6}-0.00384 y^{8} .
\end{gathered}
$$

Subcase V-A: $s=2.0$

$$
\begin{gathered}
A_{1}=0.58941, A_{2}=0.09589, \widetilde{u}(y)=0.17415-0.11625 y^{2}-0.05790 y^{4}, \\
\widetilde{E}(y)=0.01026-0.14885 y^{2}+0.24491 y^{4}-0.02692 y^{6}-0.00671 y^{8} .
\end{gathered}
$$

Subcase VI-A: $s=2.5$

$$
\begin{gathered}
A_{1}=0.48256, A_{2}=0.13156, \widetilde{u}(y)=0.12869-0.06805 y^{2}-0.06064 y^{4}, \\
\widetilde{E}(y)=0.01814-0.25859 y^{2}+0.40646 y^{4}-0.02063 y^{6}-0.00919 y^{8} .
\end{gathered}
$$

Subcase VII-A: $s=3.0$

$$
\begin{gathered}
A_{1}=0.40103, A_{2}=0.15569, \widetilde{u}(y)=0.09692-0.03661 y^{2}-0.06031 y^{4}, \\
\widetilde{E}(y)=0.02637-.37298 y^{2}+0.57384 y^{4}-0.01325 y^{6}-0.01091 y^{8} .
\end{gathered}
$$

Subcase VIII-A: $s=3.5$

$$
\begin{gathered}
A_{1}=0.33737, A_{2}=0.16909, \widetilde{u}(y)=0.07454-0.01644 y^{2}-0.05809 y^{4}, \\
\widetilde{E}(y)=0.03458-0.48712 y^{2}+0.74102 y^{4}-0.00669 y^{6}-0.01181 y^{8} .
\end{gathered}
$$

\section{Subcase IX-A: $s=4.0$}

$A_{1}=0.28681, A_{2}=0.17409, \widetilde{u}(y)=0.05853-0.00369 y^{2}-0.05484 y^{4}$,

$$
\widetilde{E}(y)=0.04251-0.59738 y^{2}+0.90306 y^{4}-0.00162 y^{6}-0.01203 y^{8} \text {. }
$$

Subcase X-A: $s=4.5$

$$
\begin{gathered}
A_{1}=0.24611, A_{2}=0.17310, \widetilde{u}(y)=0.04685+0.00426 y^{2}-0.05111 y^{4}, \\
\widetilde{E}(y)=0.04998-0.70129 y^{2}+1.05636 y^{4}+0.00196 y^{6}-0.01175 y^{8} .
\end{gathered}
$$

Subcase XI-A: $s=5$

$$
\begin{gathered}
A_{1}=0.21296, A_{2}=0.16817, \widetilde{u}(y)=0.03816+0.00908 y^{2}-0.04724 y^{4}, \\
\widetilde{E}(y)=0.05688-0.79742 y^{2}+1.19867 y^{4}+0.00429 y^{6}-0.01116 y^{8} .
\end{gathered}
$$

Case B: $F=s=1$ 
By considering the obtained values of $A_{i}$ 's our approximate solution and Residual for the following subcases, respectively, become,

Subcase I-B: $M=1$

$$
\begin{gathered}
A_{1}=0.94689, A_{2}=0.02424, \widetilde{u}(y)=0.32392-0.28656 y^{2}-0.03736 y^{4}, \\
\widetilde{E}(y)=-0.00195+0.02389 y^{2}-0.02055 y^{4}-0.02141 y^{6}-0.00139 y^{8}
\end{gathered}
$$

Subcase II-B: $M=2$

$$
\begin{gathered}
A_{1}=0.89436, A_{2}=0.01808, \widetilde{u}(y)=0.16841-0.15175 y^{2}-0.01666 y^{4}, \\
\widetilde{E}(y)=-0.00026+0.00289 y^{2}-0.00075 y^{4}-0.00506 y^{6}-0.00028 y^{8} .
\end{gathered}
$$

Subcase III-B: $M=3$

$$
\begin{gathered}
A_{1}=0.87397, A_{2}=0.01729, \widetilde{u}(y)=0.11386-0.10325 y^{2}-0.01061 y^{4}, \\
\widetilde{E}(y)=0.00001-0.0005 y^{2}+0.00236 y^{4}-0.00219 y^{6}-0.00011 y^{8} .
\end{gathered}
$$

Subcase IV-B: $M=4$

$$
A_{1}=0.86312, A_{2}=0.01724 \text {. }
$$

Via inserting these amounts the approximate solution and Residual, respectively, become as below,

$$
\begin{gathered}
\widetilde{u}(y)=0.08601-0.07825 y^{2}-0.00776 y^{4} \\
\widetilde{E}(y)=0.00009-0.00141 y^{2}+0.00297 y^{4}-0.00121 y^{6}-0.00006 y^{8}
\end{gathered}
$$

Subcase V-B: $M=5$

$$
\begin{gathered}
A_{1}=0.85638, A_{2}=0.01736, \widetilde{u}(y)=0.06911-0.06300 y^{2}-0.00611 y^{4}, \\
\widetilde{E}(y)=0.00011-0.00163 y^{2}+0.00299 y^{4}-0.00077 y^{6}-0.00004 y^{8} .
\end{gathered}
$$

Subcase VI-B: $M=6$

$$
\begin{gathered}
A_{1}=0.85179, A_{2}=0.01746, \widetilde{u}(y)=0.05777-0.05273 y^{2}-0.00504 y^{4}, \\
\widetilde{E}(y)=0.00011-0.00164 y^{2}+0.00284 y^{4}-0.00053 y^{6}-0.00003 y^{8} .
\end{gathered}
$$

Subcase VII-B: $M=7$

$$
\begin{gathered}
A_{1}=0.84846, A_{2}=0.01758, \widetilde{u}(y)=0.04962-0.04533 y^{2}-0.00428 y^{4}, \\
\widetilde{E}(y)=0.00011-0.00159 y^{2}+0.00266 y^{4}-0.00039 y^{6}-0.00002 y^{8} .
\end{gathered}
$$

Subcase VIII-B: $M=8$

$$
\begin{gathered}
A_{1}=0.84593, A_{2}=0.01768, \widetilde{u}(y)=0.04349-0.03976 y^{2}-0.00373 y^{4}, \\
\widetilde{E}(y)=0.00010-0.00151 y^{2}+0.00247 y^{4}-0.00029 y^{6}-0.00001 y^{8} .
\end{gathered}
$$


Subcase IX-B: $M=9$

$$
\begin{gathered}
A_{1}=0.84395, A_{2}=0.01778, \widetilde{u}(y)=0.03870-0.03541 y^{2}-0.00329 y^{4}, \\
\widetilde{E}(y)=0.00009-0.00142 y^{2}+0.00229 y^{4}-0.00023 y^{6}-0.00001 y^{8} .
\end{gathered}
$$

Subcase X-B: $M=10$

$$
\begin{gathered}
A_{1}=0.8423, A_{2}=0.01786, \widetilde{u}(y)=0.03487-0.03191 y^{2}-0.00296 y^{4}, \\
\widetilde{E}(y)=0.00009-0.00134 y^{2}+0.00214 y^{4}-0.00019 y^{6}-0.000009 y^{8} .
\end{gathered}
$$

Case C: $M=s=1$

By considering the obtained values of $A_{i}$ 's our approximate solution and Residual for the following subcases, respectively, become,

Subcase I-C: $F=0$

$$
\begin{gathered}
A_{1}=0.82752, A_{2}=0.01889, \widetilde{u}(y)=0.35190-0.32337 y^{2}-0.02853 y^{4}, \\
\widetilde{E}(y)=0.00136-0.01902 y^{2}+0.02853 y^{4} .
\end{gathered}
$$

Subcase II-C: $F=1$

$$
\begin{gathered}
A_{1}=0.94689, A_{2}=0.02424, \widetilde{u}(y)=0.32392-.28656 y^{2}-0.037356 y^{4}, \\
\widetilde{E}(y)=-0.00195+0.02389 y^{2}-0.02055 y^{4}-0.02141 y^{6}-0.00139 y^{8} .
\end{gathered}
$$

Subcase III-C: $F=2$

$$
\begin{gathered}
A_{1}=1.02545, A_{2}=0.04426, \widetilde{u}(y)=0.30273-0.25892 y^{2}-0.04381 y^{4}, \\
\widetilde{E}(y)=-0.00387+0.04668 y^{2}-0.03721 y^{4}-0.04538 y^{6}-0.00384 y^{8} .
\end{gathered}
$$

Subcase IV-C: $F=3$

$$
\begin{gathered}
A_{1}=1.0822, A_{2}=0.06642, \widetilde{u}(y)=0.28583-0.23703 y^{2}-0.04880 y^{4}, \\
\widetilde{E}(y)=-0.00497+0.05791 y^{2}-0.03605 y^{4}-0.06940 y^{6}-0.00714 y^{8} .
\end{gathered}
$$

Subcase V-C: $F=4$

$$
\begin{gathered}
A_{1}=1.12564, A_{2}=0.08742, \widetilde{u}(y)=0.27185-0.21905 y^{2}-0.05279 y^{4}, \\
\widetilde{E}(y)=-0.00555+0.06191 y^{2}-0.02433 y^{4}-0.09252 y^{6}-0.01115 y^{8} .
\end{gathered}
$$

Subcase VI-C: $F=5$

$$
\begin{gathered}
A_{1}=1.16012, A_{2}=0.10639, \widetilde{u}(y)=.25999-.20391 y^{2}-0.05608 y^{4}, \\
\widetilde{E}(y)=-0.00579+0.06112 y^{2}-0.00602 y^{4}-.11435 y^{6}-0.15724 y^{8} .
\end{gathered}
$$

Subcase VII-C: $F=6$

$$
\begin{gathered}
A_{1}=1.18826, A_{2}=0.12323, \widetilde{u}(y)=0.24974-.19091 y^{2}-0.05883 y^{4}, \\
\widetilde{E}(y)=-0.00578+0.05706 y^{2}+0.01647 y^{4}-.13478 y^{6}-0.02077 y^{8} .
\end{gathered}
$$



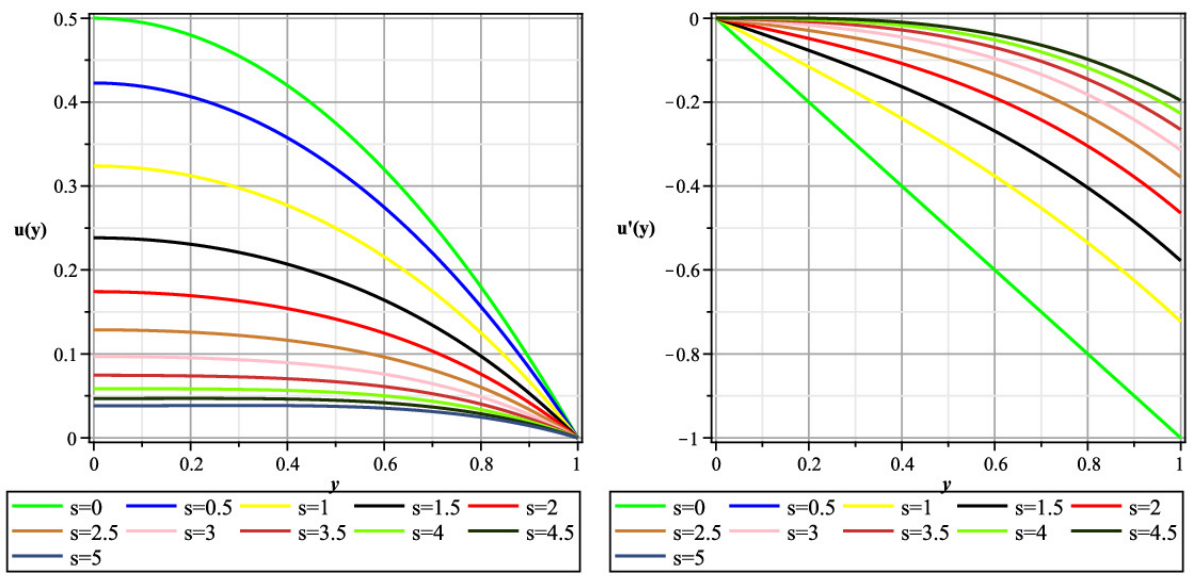

Figure 3. Profile of (left) the DBF equation (right) the derivative of DBF equation for diverse amounts of $s$ with $M=F=1$.

\section{Subcase VIII-C: $F=7$}

$$
\begin{gathered}
A_{1}=1.21169, A_{2}=0.13806, \widetilde{u}(y)=0.24074-.17957 y^{2}-0.06118 y^{4}, \\
\widetilde{E}(y)=-0.00559+0.05069 y^{2}+0.04165 y^{4}-.15379 y^{6}-0.02619 y^{8} .
\end{gathered}
$$

Subcase IX-C: $F=8$

$$
\begin{gathered}
A_{1}=1.23154, A_{2}=0.15108, \widetilde{u}(y)=0.23276-.16956 y^{2}-0.06319 y^{4}, \\
\widetilde{E}(y)=-0.00528+0.04267 y^{2}+0.06854 y^{4}-.17145 y^{6}-0.03195 y^{8} .
\end{gathered}
$$

Subcase X-C: $F=9$

$$
\begin{gathered}
A_{1}=1.24857, A_{2}=0.16251, \widetilde{u}(y)=0.22559-.16063 y^{2}-0.06495 y^{4}, \\
\widetilde{E}(y)=-0.00486+0.03344 y^{2}+0.09648 y^{4}-.18781 y^{6}-0.03797 y^{8} .
\end{gathered}
$$

Subcase XI-C: $F=10$

$$
\begin{gathered}
A_{1}=1.26332, A_{2}=0.17254, \widetilde{u}(y)=0.21910-0.15260 y^{2}-0.06649 y^{4}, \\
\widetilde{E}(y)=-0.00438+0.02334 y^{2}+.12502 y^{4}-.20296 y^{6}-0.04422 y^{8} .
\end{gathered}
$$

4.2. Application of the OG-HAM for FTD equation. We utilize the offered method (OG-HAM) to study the FTD where given by Eq. (2.8) and (2.9).

Theorem 4.2. Following the $O G-H A M$ formulation, we have:

$$
\mathcal{K}(\theta(x))=\frac{d^{2} \theta(x)}{d x^{2}}, \quad \mathcal{M}(\theta(x))=\beta\left(\frac{d \theta}{d x}\right)^{2}+\beta \theta \frac{d^{2} \theta(x)}{d x^{2}}-\psi^{2},
$$

with the initial condition:

$$
\theta^{\prime}(0)=0, \quad \theta(1)=1 .
$$



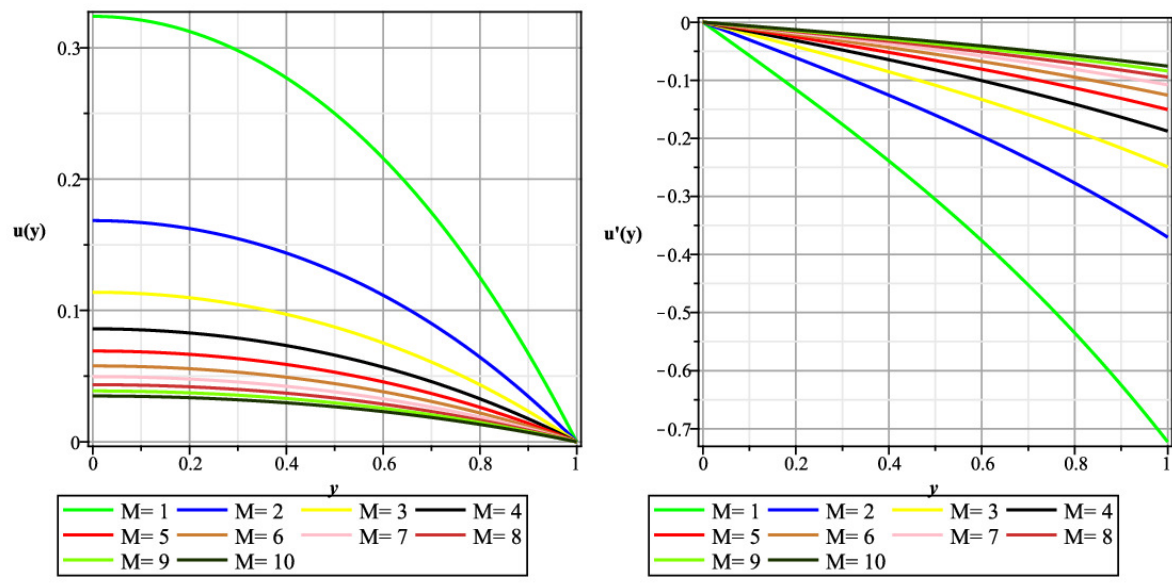

Figure 4. Profile of (left) the DBF equation (right) the derivative of DBF equation for diverse amounts of $M$ with $s=F=1$.
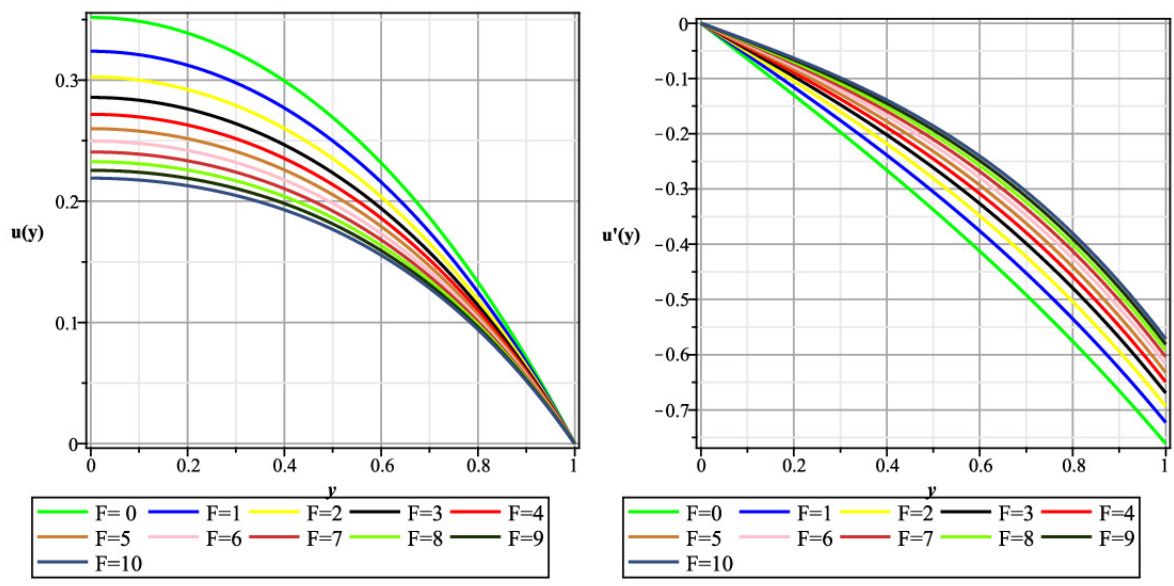

Figure 5. Profile of (left) the DBF equation (right) the derivative of DBF equation for diverse amounts of $F$ with $s=M=1$.

With the second order approximate solution by OHAM for, $q=1$, is;

$$
\widetilde{\theta}\left(x, A_{1}, A_{2}\right)=\theta_{0}(x)+\theta_{1}\left(x, A_{1}\right)+\theta_{2}\left(x, A_{1}, A_{2}\right) .
$$

For the domain $\Sigma=[a, b]=[0,1]$, the residual will be utilized as

$$
\mathcal{E}\left(x, A_{1}, A_{2}\right)=\frac{d^{2} \widetilde{\theta}\left(x, A_{1}, A_{2}\right)}{d x^{2}}+\beta \frac{d}{d x}\left(\widetilde{\theta}\left(x, A_{1}, A_{2}\right) \frac{d^{2} \widetilde{\theta}\left(x, A_{1}, A_{2}\right)}{d x^{2}}\right)-\psi^{2} .
$$

Zeroth-order problem defined via Eq. (3.5) is as

$$
\frac{d^{2} \theta_{0}(x)}{d x^{2}}=0, \quad \theta_{0}^{\prime}(0)=0, \quad \theta_{0}(1)=1
$$

from which we can get

$$
\theta_{0}(x)=1
$$



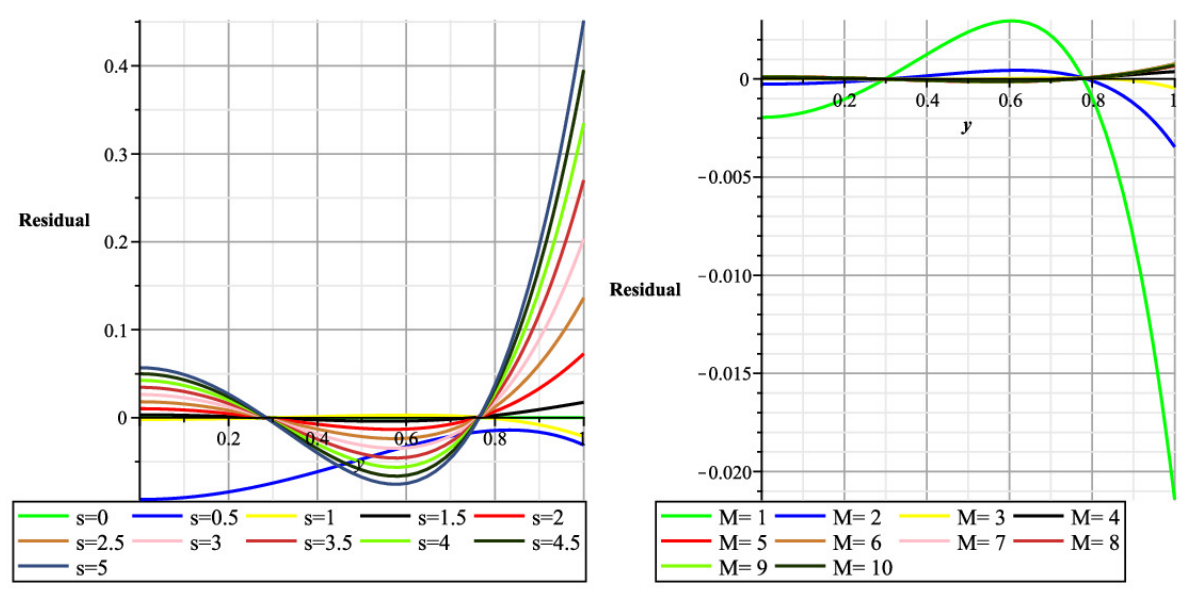

Figure 6. Profile of (left) the Residual of DBF equation for diverse amounts of $s$ with $F=M=1$ (right) the Residual of DBF equation for various values of $M$ when $s=F=1$.

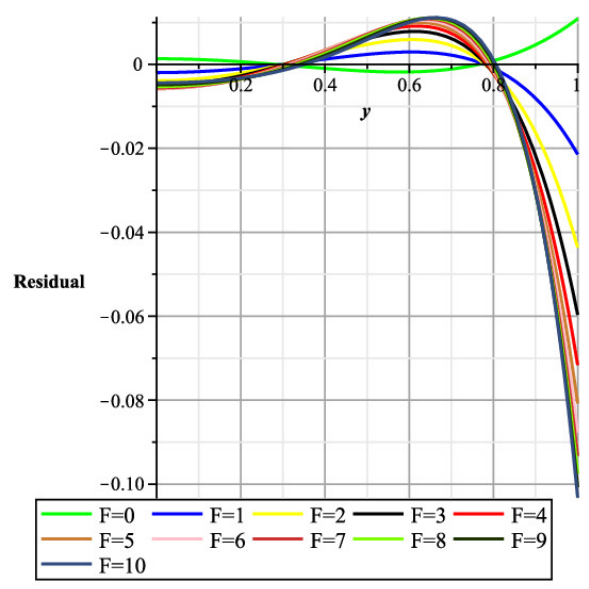

FiguRE 7. Profile of the Residual of DBF equation for diverse amounts of $F$ with $s=M=1$.

First-order problem defined via Eq. (3.8) is as:

$\theta_{1}^{\prime \prime}\left(x, A_{1}\right)=\left(1-A_{1}\right) \theta_{0}^{\prime \prime}(x)-A_{1} \beta\left(\theta_{0}^{\prime 2}(x)+\theta_{0}(x) \theta_{0}^{\prime \prime}(x)\right)+A_{1} \psi^{2}, \theta_{1}^{\prime}(0)=0, \theta_{1}(1)=0$.

The solution will can be written as below:

$$
\theta_{1}\left(x, A_{1}\right)=\frac{1}{2} A_{1} \psi^{2}\left(x^{2}-1\right)
$$

Second-order problem given by Eq. (3.9):

$\theta_{2}^{\prime \prime}\left(x, A_{1}, A_{2}\right)=\left(1-A_{1}\right) \theta_{1}^{\prime \prime}\left(x, A_{1}\right)+A_{1} \psi^{2}-C_{2} \theta_{0}^{\prime \prime}(x)+A_{2} \beta\left(\theta_{0}(x) \theta_{0}^{\prime \prime}(x)+\theta_{0}^{\prime}(x)\right)$

$-A_{1} \beta\left(\theta_{1}\left(x, A_{1}\right) \theta_{0}^{\prime \prime}(x)+\theta_{0}(x) \theta_{1}^{\prime \prime}\left(x, A_{1}\right)+2 \theta_{0}^{\prime}(x) \theta_{1}^{\prime}\left(x, A_{1}\right)\right), \quad \theta_{2}^{\prime}(0)=0, \quad \theta_{2}(1)=0$. 
The solution becomes:

$$
\begin{aligned}
\theta_{2}\left(x, A_{1}, A_{2}\right)= & 0.041667 A_{1}^{2} \psi^{4} x^{4}+\frac{1}{2} \psi^{2} x^{2}\left(A_{2}-(\beta+1) A_{1}^{2}+A_{1}-0.5 A_{1}^{2} \psi^{2}\right) \\
& +0.20833 A_{1}^{2} \psi^{4}-\frac{1}{2} \psi^{2}\left(A_{1}+A_{2}-(\beta+1) A_{1}^{2}\right) .
\end{aligned}
$$

Using Eqs. (4.82), (4.84) and (4.86), the second order approximate solution by OHAM for, $q=1$, is;

$$
\widetilde{\theta}\left(x, A_{1}, A_{2}\right)=\theta_{0}(x)+\theta_{1}\left(x, A_{1}\right)+\theta_{2}\left(x, A_{1}, A_{2}\right) .
$$

Following the procedure described in Section 3 on the domain $\Sigma=[a, b]=[0,1]$, utilizing the residual,

$$
\mathcal{E}\left(x, A_{1}, A_{2}\right)=\frac{d^{2} \widetilde{\theta}\left(x, A_{1}, A_{2}\right)}{d x^{2}}+\beta \frac{d}{d x}\left(\widetilde{\theta}\left(x, A_{1}, A_{2}\right) \frac{d^{2} \widetilde{\theta}\left(x, A_{1}, A_{2}\right)}{d x^{2}}\right)-\psi^{2} .
$$

Case A: $\beta=0.1$

Via considering the obtained amounts of $A_{i}$ 's our approximate solution and Residual for the following subcases, respectively, become,

Subcase I-A: $\psi=0.1$

$$
\begin{aligned}
& A_{1}=0.77436, A_{2}=0.01922, \widetilde{\theta}(x)=0.99547+0.00453 x^{2}+0.000002 x^{4}, \\
& \widetilde{E}(x)=6.094 \times 10^{-10}-6.895 \times 10^{-9} x^{2}+8.945 \times 10^{-9} x^{4}+1.748 \times 10^{-11} x^{6} .
\end{aligned}
$$

Subcase II-A: $\psi=0.2$

$$
\begin{aligned}
& A_{1}=0.77161, A_{2}=0.01786, \widetilde{\theta}(x)=0.98208+0.01789 x^{2}+0.00004 x^{4}, \\
& \widetilde{E}(x)=3.069 \times 10^{-8}-3.867 \times 10^{-7} x^{2}+5.420 \times 10^{-7} x^{4}+4.411 \times 10^{-9} x^{6} .
\end{aligned}
$$

Subcase III-A: $\psi=0.3$

$$
\begin{gathered}
A_{1}=0.76710, A_{2}=0.01579, \widetilde{\theta}(x)=0.93123+0.06815 x^{2}+0.00062 x^{4}, \\
\widetilde{E}(x)=2.721 \times 10^{-7}-0.000 x^{2}+5.620 \times 10^{-6} x^{4}+1.104 \times 10^{-7} x^{6} .
\end{gathered}
$$

Subcase IV-A: $\psi=0.4$

$$
\begin{gathered}
A_{1}=0.76078, A_{2}=0.01331, \widetilde{\theta}(x)=0.96037+0.03943 x^{2}+0.00019 x^{4}, \\
\widetilde{E}(x)=0.000001-0.000019 x^{2}+0.00003 x^{4}+0.000001 x^{6} .
\end{gathered}
$$

Subcase V-A: $\psi=0.5$

$$
\begin{gathered}
A_{1}=0.75278, A_{2}=0.01076, \widetilde{\theta}(x)=0.89576+0.10277 x^{2}+0.00148 x^{4}, \\
\widetilde{E}(x)=0.000004-0.00006 x^{2}+0.00009 x^{4}+0.000006 x^{6} .
\end{gathered}
$$

Case B: $\beta=0.2$ 
Via considering the obtained amounts of $A_{i}$ 's our approximate solution and Residual for the following subcases, respectively, become,

Subcase I-B: $\psi=0.1$

$$
\begin{aligned}
& A_{1}=0.58945, A_{2}=0.07023, \widetilde{\theta}(x)=0.99585+0.00415 x^{2}+0.000001 x^{4}, \\
& \widetilde{E}(x)=-1.714 \times 10^{-9}-7.084 \times 10^{-10} x^{2}+2.159 \times 10^{-8} x^{4}+1.174 \times 10^{-11} x^{6} .
\end{aligned}
$$

Subcase II-B: $\psi=0.2$

$$
\begin{aligned}
& A_{1}=0.58918, A_{2}=0.06702, \widetilde{\theta}(x)=0.98354+0.01644 x^{2}+0.00002 x^{4}, \\
& \widetilde{E}(x)=7.156 \times 10^{-8}-9.398 \times 10^{-7} x^{2}+1.350 \times 10^{-6} x^{4}+2.999 \times 10^{-9} x^{6} .
\end{aligned}
$$

Subcase III-B: $\psi=0.3$

$$
\begin{aligned}
& A_{1}=0.58902, A_{2}=0.06177, \widetilde{\theta}(x)=0.96353+0.03636 x^{2}+0.00012 x^{4}, \\
& \widetilde{E}(x)=7.173 \times 10^{-7}-1.003 \times 10^{-6} x^{2}+1.5 \times 10^{-5} x^{4}+7.678 \times 10^{-8} x^{6} .
\end{aligned}
$$

Subcase IV-B: $\psi=0.4$

$$
\begin{gathered}
A_{1}=0.58853, A_{2}=0.05509, \widetilde{\theta}(x)=0.93653+0.06310 x^{2}+0.00037 x^{4}, \\
\widetilde{E}(x)=0.000004-0.000054 x^{2}+0.00008 x^{4}+7.644 \times 10^{-7} x^{6} .
\end{gathered}
$$

Subcase V-B: $\psi=0.5$

$$
\begin{gathered}
A_{1}=0.58757, A_{2}=0.04754, \widetilde{\theta}(x)=0.90345+0.09565 x^{2}+0.00089 x^{4}, \\
\widetilde{E}(x)=0.00001-0.00019 x^{2}+0.00029 x^{4}+0.000005 x^{6} .
\end{gathered}
$$

Case C: $\beta=0.3$

Via considering the obtained amounts of $A_{i}$ 's our approximate solution and Residual for the following subcases, respectively, become,

Subcase I-C: $\psi=0.1$

$$
\begin{gathered}
A_{1}=0.42783, A_{2}=0.15016, \widetilde{\theta}(x)=0.99616+0.00383 x^{2}+7.627 \times 10^{-7} x^{4}, \\
\widetilde{E}(x)=-3.456 \times 10^{-9}+9.256 \times 10^{-9} x^{2}+1.869 \times 10^{-8} x^{4}+4.886 \times 10^{-12} x^{6} .
\end{gathered}
$$

Subcase II-C: $\psi=0.2$

$$
\begin{aligned}
& A_{1}=0.42956, A_{2}=0.14465, \widetilde{\theta}(x)=0.98478+0.01520 x^{2}+0.00001 x^{4}, \\
& \widetilde{E}(x)=6.751 \times 10^{-8}-8.483 \times 10^{-7} x^{2}+0.000001 x^{4}+1.271 \times 10^{-9} x^{6} .
\end{aligned}
$$

Subcase III-C: $\psi=0.3$

$$
\begin{gathered}
A_{1}=0.43300, A_{2}=0.13527, \widetilde{\theta}(x)=0.96623+0.03371 x^{2}+0.00006 x^{4}, \\
\widetilde{E}(x)=6.439 \times 10^{-7}-0.000009 x^{2}+0.00001 x^{4}+3.364 \times 10^{-8} x^{6} .
\end{gathered}
$$


Subcase IV-C: $\psi=0.4$

$$
\begin{gathered}
A_{1}=0.43730, A_{2}=0.12320, \widetilde{\theta}(x)=0.94108+0.05871 x^{2}+0.00020 x^{4}, \\
\widetilde{E}(x)=0.000004-0.0000503 x^{2}+0.00008 x^{4}+3.495 \times 10^{-7} x^{6} .
\end{gathered}
$$

Subcase V-C: $\psi=0.5$

$$
\begin{gathered}
A_{1}=0.44213, A_{2}=0.10923, \widetilde{\theta}(x)=0.91012+0.08937 x^{2}+0.00051 x^{4}, \\
\widetilde{E}(x)=0.000014-0.00019 x^{2}+0.00028 x^{4}+0.0000022 x^{6} .
\end{gathered}
$$

Case D: $\beta=0.4$

Via considering the obtained amounts of $A_{i}$ 's our approximate solution and Residual for the following subcases, respectively, become,

Subcase I-D: $\psi=0.1$

$$
\begin{gathered}
A_{1}=0.27204, A_{2}=0.27237, \widetilde{\theta}(x)=0.99644+0.00356 x^{2}+3.084 \times 10^{-7} x^{4}, \\
\widetilde{E}(x)=-2.489 \times 10^{-9}+8.119 \times 10^{-9} x^{2}+1.009 \times 10^{-8} x^{4}+1.065 \times 10^{-12} x^{6} .
\end{gathered}
$$

Subcase II-D: $\psi=0.2$

$$
\begin{aligned}
& A_{1}=0.27639, A_{2}=.26273, \widetilde{\theta}(x)=0.98585+0.01414 x^{2}+0.000005 x^{4}, \\
& \widetilde{E}(x)=4.506 \times 10^{-8}-5.083 \times 10^{-7} x^{2}+6.605 \times 10^{-7} x^{4}+2.905 \times 10^{-10} x^{6} .
\end{aligned}
$$

Subcase III-D: $\psi=0.3$

$$
\begin{gathered}
A_{1}=0.28412, A_{2}=0.24651, \widetilde{\theta}(x)=0.96856+0.03141 x^{2}+0.000027 x^{4}, \\
\widetilde{E}(x)=3.722 \times 10^{-7}-0.000005 x^{2}+0.000008 x^{4}+8.313 \times 10^{-9} x^{6} .
\end{gathered}
$$

Subcase IV-D: $\psi=0.4$

$$
\begin{gathered}
A_{1}=0.29388, A_{2}=0.22588, \tilde{\theta}(x)=0.94504+.054865 x^{2}+0.00009 x^{4}, \\
\widetilde{E}(x)=0.000002-0.000031 x^{2}+0.000046 x^{4}+9.505 \times 10^{-8} x^{6} .
\end{gathered}
$$

Subcase V-D: $\psi=0.5$

$$
\begin{gathered}
A_{1}=0.30508, A_{2}=0.20216, \tilde{\theta}(x)=0.91596+0.08379 x^{2}+0.00024 x^{4}, \\
\widetilde{E}(x)=0.000009-0.000122 x^{2}+0.00018 x^{4}+6.579 \times 10^{-7} x^{6} .
\end{gathered}
$$

Case E: $\beta=0.5$

Via considering the obtained amounts of $A_{i}$ 's our approximate solution and Residual for the following subcases, respectively, become,

Subcase I-E: $\psi=0.1$

$$
\begin{gathered}
A_{1}=0.08958, A_{2}=0.49809, \widetilde{\theta}(x)=0.99667+0.00333 x^{2}+3.344 \times 10^{-8} x^{4}, \\
\widetilde{E}(x)=-1.055 \times 10^{-7}+5.271 \times 10^{-7} x^{2}+1.334 \times 10^{-9} x^{4}+1.565 \times 10^{-14} x^{6} .(4.130)
\end{gathered}
$$



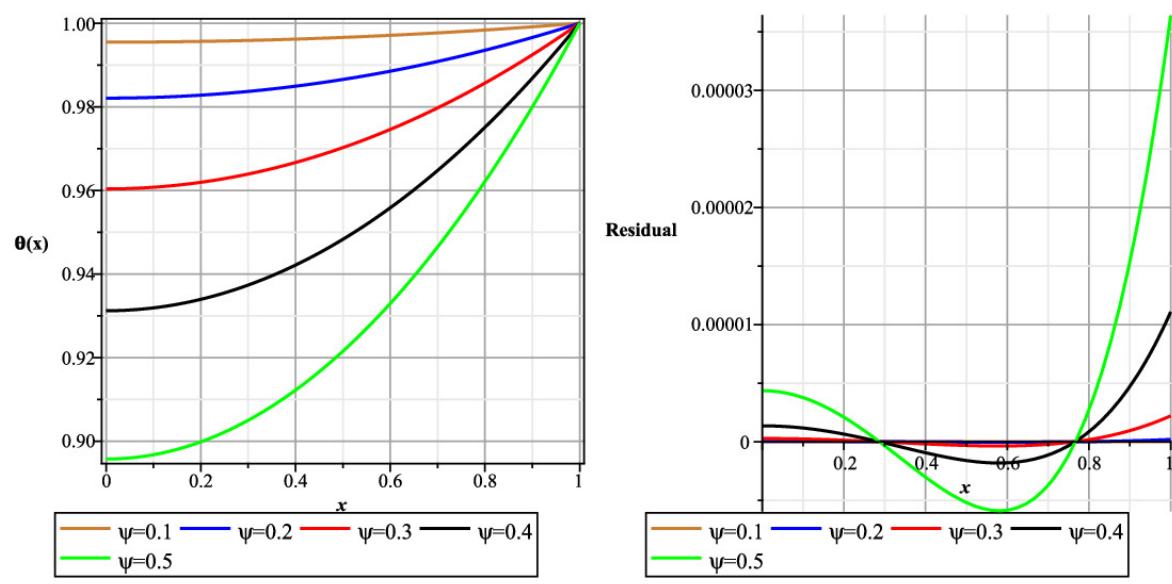

Figure 8. Profile of (left) the FTD (right) the Residual of FTD for diverse amounts of $\psi$ with $\beta=0.1$.

Subcase II-E: $\psi=0.2$

$$
\begin{gathered}
A_{1}=0.06414, A_{2}=0.53874, \widetilde{\theta}(x)=0.98678+0.01322 x^{2}+2.743 \times 10^{-7} x^{4}, \\
\widetilde{E}(x)=-5.107 \times 10^{-8}+2.366 \times 10^{-7} x^{2}+4.339 \times 10^{-8} x^{4}+1.053 \times 10^{-12} x^{6} .
\end{gathered}
$$

Subcase III-E: $\psi=0.3$

$$
\begin{aligned}
& A_{1}=0.09273, A_{2}=0.48129, \widetilde{\theta}(x)=0.97059+0.02941 x^{2}+0.000003 x^{4}, \\
& \widetilde{E}(x)=4.828 \times 10^{-8}-6.782 \times 10^{-7} x^{2}+0.000001 x^{4}+1.179 \times 10^{-10} x^{6} .
\end{aligned}
$$

Subcase IV-E: $\psi=0.4$

$$
\begin{gathered}
A_{1}=0.12207, A_{2}=0.42279, \widetilde{\theta}(x)=0.94851+0.05147 x^{2}+0.000016 x^{4}, \\
\widetilde{E}(x)=4.496 \times 10^{-7}-0.000006 x^{2}+0.000009 x^{4}+3.5363 \times 10^{-9} x^{6} .
\end{gathered}
$$

Subcase V-E: $\psi=0.5$

$$
\begin{gathered}
A_{1}=0.15009, A_{2}=0.36709, \widetilde{\theta}(x)=0.92110+0.07883 x^{2}+0.00006 x^{4}, \\
\widetilde{E}(x)=0.000003-0.00004 x^{2}+0.00006 x^{4}+4.818 \times 10^{-8} x^{6} .
\end{gathered}
$$

\section{Numerical Solution via OGHAM}

Now, considering the results obtained by OGHAM in the form of tables and figures. The simulation comparisons according to effects of free parameters, namely, $s, F$ and $M$ in DBF momentum equation and $\beta$ and $\psi$ in Fin temperature distribution by Exact solutions, THAM solutions and OGHAM solutions are shown. In addition, the errors and residuals are as well as presented in this part. The simulation solutions for the nonlinear second order boundary value problems are 

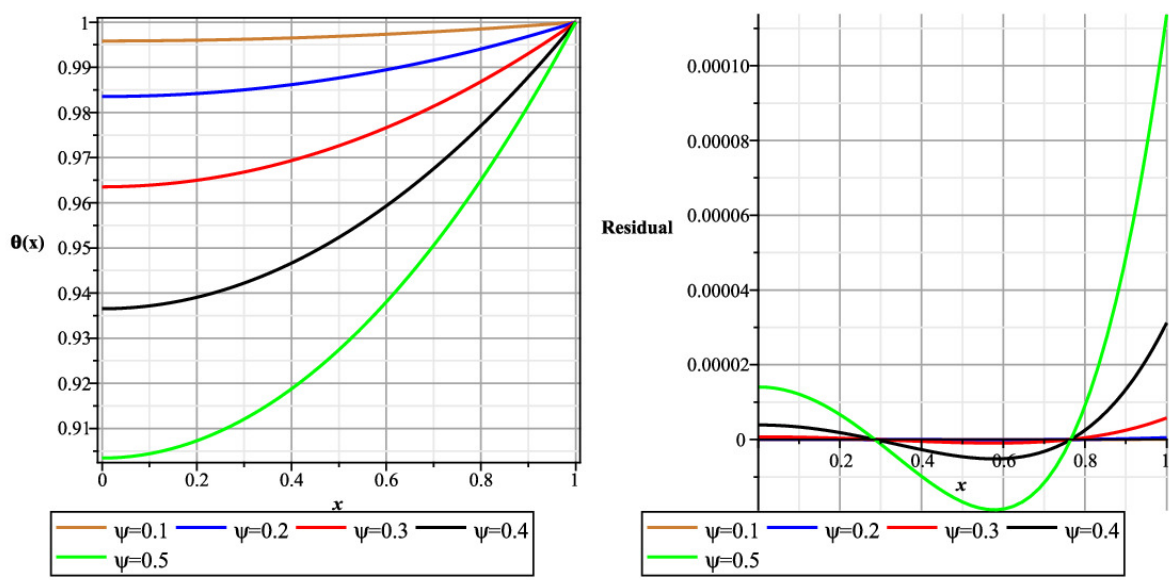

Figure 9. Profile of (left) the FTD (right) the Residual of FTD for diverse amounts of $\psi$ with $\beta=0.2$.
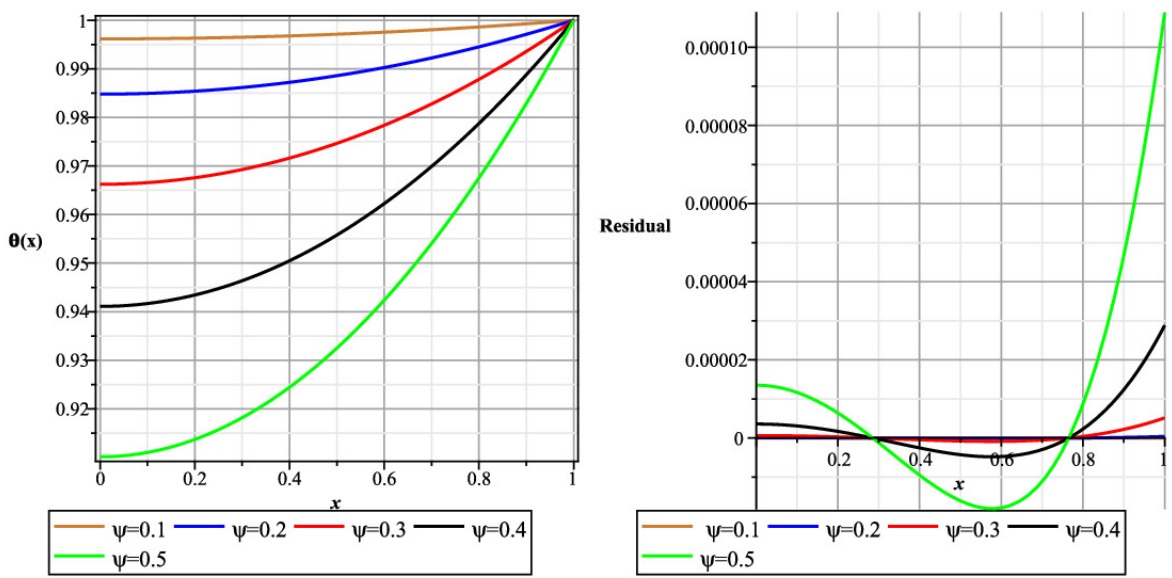

FiguRE 10. Profile of (left) the FTD (right) the Residual of FTD for diverse amounts of $\psi$ with $\beta=0.3$.

received. Three diverse schemes namely, THAM, and OGHAM are utilized for the solutions of nonlinear BVPs. The results of the DBF equation and the residual of it and also results of the fin and the residual of it obtained from the nonlinear BVPs are compared numerically in Tables 1-8 for diverse amounts of embedded parameters of $s, F, M$ and $\beta, \psi$. All consequences for the nonlinear BVPs are demonstrated in the $x$-coordinate only for as selected domain $x \in[0,1]$. As well as, the Residual of DBF for diverse amounts, namely, the viscosity ratio $(M)$, the Forchheimer number $(F)$ and the porous medium shape parameter $(s)$ are depicted in Figures 3-7. Also, in Figures 8-12, FTD for two parameters the thermo-geometric fin parameter $(\psi)$ and the thermal conductivity parameter $(\beta)$ are discussed. It can be vividly viewed from Figures 3-5 that an exponential rate of convergence is obtained. Figures 3-5, show that comparison the DBF equation and derivative of it when the porous medium shape parameter $s$, the viscosity 

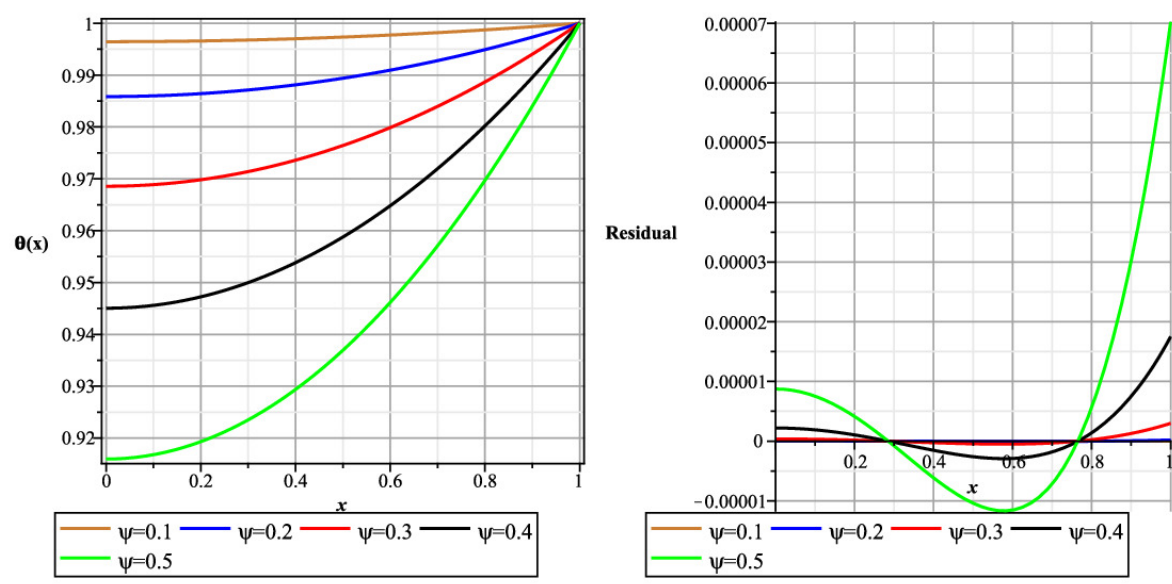

FiguRE 11. Profile of (left) the FTD (right) the Residual of FTD for diverse amounts of $\psi$ with $\beta=0.4$.
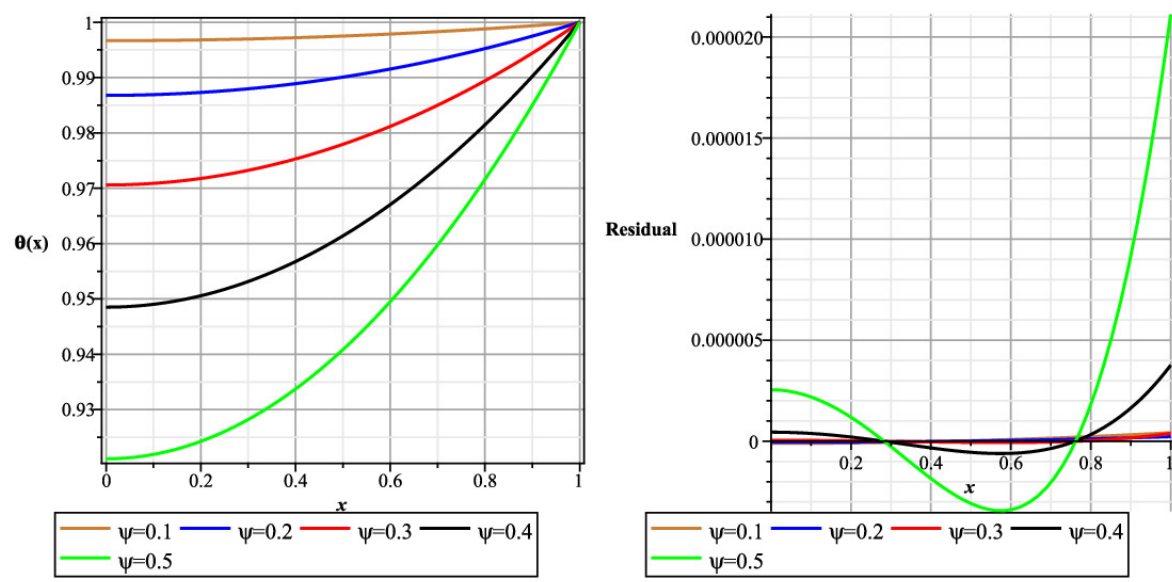

FiguRE 12. Profile of (left) the FTD (right) the Residual of FTD for diverse amounts of $\psi$ with $\beta=0.5$.

ratio $M$ and the Forchheimer number $F$, respectively, change. Also, figures 6 and 7 show that the Residual of DBF equation, when the porous medium shape parameter $s$, the viscosity ratio $M$ and the Forchheimer number $F$, respectively, change. For different the thermal conductivity and the thermo-geometric fin parameters, figures 8-12 are depicted. A comparison between present method results and similar results of literature for $u(0)$ and $u^{\prime}(1)$ for diverse amounts of $s$ with $M=F=1$ in Table 1 , diverse amounts of $M$ with $s=F=1$ in Table 2 and diverse amounts of $F$ with $s=M=1$ in Table 3, for DBF equation are given. Finally, comparisons of the residual of FTD when $\beta$ is changed in Tables 4- 8 are given. In made works by some scholars one can see a comparison between OGHAM and ADM ([62]), HAM ([1]) and some other methods presented at $([10,37,49,52,57])$. The obtained errors by our show the efficiency of method 
in works offered in the literature. Also, the error of equations has been calculated by replacing the solutions in the method is near $10^{-10}$. Of course, the more distance of the initial point results in the greater discrepancy. So, the error of obtained solution has been improved by decreasing the step length.

TABLE 1. A comparison between current method results and similar results of literature for $u(0)$ and $u^{\prime}(1)$ for diverse amounts of $s$ with $M=F=1$ for DBF equation.

\begin{tabular}{|c|c|c|c|c|c|c|}
\hline & \multicolumn{2}{|c|}{$\bar{u} u(0)$} & \multicolumn{4}{|c|}{$\overline{u^{\prime}(1)}$} \\
\hline$s$ & Exact [1] & THAM [56] & OHAM & Exact [1] & THAM [56] & OHAM \\
\hline 0.0 & 0.50000000 & 0.50000000 & 0.49999999 & -1.00000000 & -1.00000000 & -0.99999999 \\
\hline 0.5 & 0.42268539 & 0.42268512 & 0.42280349 & -0.88064312 & -0.88064302 & -0.88266829 \\
\hline 1.0 & 0.32384748 & 0.32384737 & 0.32391684 & -0.72123255 & -0.72123150 & -0.72255188 \\
\hline 1.5 & 0.23838512 & 0.23838511 & 0.23829315 & -0.57910415 & -0.57910403 & -0.57776604 \\
\hline 2.0 & 0.17443254 & 0.17443249 & 0.17414743 & -0.46912865 & -0.46912838 & -0.46409652 \\
\hline 2.5 & 0.12917334 & 0.12917323 & 0.12869561 & -0.38790266 & -0.38790262 & -0.37867709 \\
\hline 3.0 & 0.09756501 & 0.09756552 & 0.09691552 & -0.32802775 & -0.32802769 & -0.31445019 \\
\hline 3.5 & 0.07532735 & 0.07532713 & 0.07453745 & -0.28310477 & -0.28310468 & -0.26526295 \\
\hline 4.0 & 0.05942133 & 0.05942103 & 0.05852592 & -0.24857364 & -0.24857355 & -0.22673197 \\
\hline 4.5 & 0.04781481 & 0.04781437 & 0.04684759 & -0.22137069 & -0.22137051 & -0.19590678 \\
\hline 5.0 & 0.03916962 & 0.03916923 & 0.03816031 & -0.19945535 & -0.19945515 & -0.17080529 \\
\hline
\end{tabular}

\section{Conclusion}

In this paper, we considered the nonlinear second order boundary value problem. The approximate solutions of the nonlinear second-order boundary value problem achieved by applying a new analytical technique namely, OG-HAM. The result obtained from the techniques compared to numerically and graphically. The result of THAM and OG-HAM close associated with each other. The effects of model parameters are presented graphically on the DBF and DTB and residual of those. Although the method of optimal Galerkin-homotopy asymptotic is famous and applicable for various problems, in this paper it has been shown that the error of solution obtained by the method in a known equation is sizeable, at least. The fin temperature distribution has been considered in this paper. The approximate solutions of several iterations have been obtained by OGHAM and has been presented their errors. We have selected an appropriate iteration of HAM with less error in a first subinterval. Based on this obtained solution and suitable step, the OGHAM has been utilized. There is an intense distinction between the solution obtained by HAM and OGHAM. The values and differences of solutions at several points have been illustrated in the figures and tables. Also, the error of equations has been calculated by replacing the solutions 
TABLE 2. A comparison between current method results and similar results of literature for $u(0)$ and $u^{\prime}(1)$ for diverse amounts of $M$ with $s=F=1$ for DBF equation.

\begin{tabular}{|c|c|c|c|c|c|c|}
\hline & & (0) & & $\bar{u} u^{\prime}(1)$ & & \\
\hline$M$ & Exact [1] & THAM [56] & OHAM & Exact [1] & THAM [56] & OHAM \\
\hline 1.0 & 0.32384748 & 0.32384746 & 0.32391684 & -0.72123156 & -0.72123154 & -0.72255188 \\
\hline 2.0 & 0.16840089 & 0.16840085 & 0.16841012 & -0.36994359 & -0.36994358 & -0.370148202 \\
\hline 3.0 & 0.11385781 & 0.11385781 & 0.11385776 & -0.24891284 & -0.24891282 & -0.248932866 \\
\hline 4.0 & 0.08601438 & 0.08601437 & 0.08601184 & -0.18757523 & -0.18757522 & -0.187544056 \\
\hline 5.0 & 0.06911628 & 0.06911628 & 0.06911299 & -0.15049696 & -0.15049693 & -0.150449157 \\
\hline 6.0 & 0.05776862 & 0.05776862 & 0.05776516 & -0.12566013 & -0.12566011 & -0.125607334 \\
\hline 7.0 & 0.04962212 & 0.04962002 & 0.04961872 & -0.10786072 & -0.10786522 & -0.107807432 \\
\hline 8.0 & 0.04348953 & 0.04348623 & 0.04348625 & -0.09447861 & -0.09447069 & -0.094426650 \\
\hline 9.0 & 0.03870614 & 0.03870456 & 0.03870302 & -0.08405082 & -0.08403012 & -0.084000924 \\
\hline 10.0 & 0.03487080 & 0.03487001 & 0.03486799 & -0.07569623 & -0.07569257 & -0.075648636 \\
\hline
\end{tabular}

TABLE 3. A comparison between current method results and similar results of literature for $u(0)$ and $u^{\prime}(1)$ for diverse amounts of $F$ with $s=M=1$ for DBF equation.

\begin{tabular}{|c|c|c|c|c|c|c|}
\hline & $\overrightarrow{u(}$ & 0) & & $\overline{u u^{\prime}(1)}$ & & \\
\hline$F$ & Exact [1] & THAM [56] & OHAM & Exact [1] & THAM [56] & OHAM \\
\hline 0.0 & 0.35194573 & 0.35194571 & 0.35190217 & -0.76159416 & -0.76159411 & -0.76086957 \\
\hline 1.0 & 0.32384748 & 0.32384751 & 0.32391684 & -0.72123156 & -0.72123151 & -0.72255188 \\
\hline 2.0 & 0.30260920 & 0.30260920 & 0.30273429 & -0.69043361 & -0.69043358 & -0.69309711 \\
\hline 3.0 & 0.28566735 & 0.28566733 & 0.28582690 & -0.66566104 & -0.66566101 & -0.66925454 \\
\hline 4.0 & 0.27166879 & 0.27166875 & 0.27184658 & -0.64503208 & -0.64503203 & -0.64928185 \\
\hline 5.0 & 0.25980414 & 0.25980411 & 0.25998982 & -0.62741989 & -0.62741976 & -0.63213575 \\
\hline 6.0 & 0.24955327 & 0.24973977 & 0.24973978 & -0.61209816 & -0.61207546 & -0.61714215 \\
\hline 7.0 & 0.24056245 & 0.24056045 & 0.24074488 & -0.59857162 & -0.59831762 & -0.60384052 \\
\hline 8.0 & 0.23258057 & 0.23218037 & 0.23275542 & -0.58648782 & -0.58632752 & -0.59190248 \\
\hline 9.0 & 0.22542325 & 0.22522835 & 0.22558798 & -0.57558726 & -0.57543226 & -0.58108578 \\
\hline 10.0 & 0.21895122 & 0.21835155 & 0.21910401 & -0.56567351 & -0.56566052 & -0.57120674 \\
\hline
\end{tabular}

in the aforementioned method. Of course, the more distance of the initial point results in the greater discrepancy. So, the error of obtained solution has been improved by decreasing the step length. 
TABLE 4. Comparison of the Residual of FTD when $\beta=0.1$

\begin{tabular}{llllll}
\hline \hline$x$ & $\psi=0.1$ & $\psi=0.2$ & $\psi=0.3$ & $\psi=0.4$ & $\psi=0.5$ \\
\hline 0.0 & $6.09 \times 10^{-10}$ & $3.07 \times 10^{-8}$ & $2.73 \times 10^{-7}$ & $3.89 \times 10^{-6}$ & $1.41 \times 10^{-5}$ \\
0.1 & $5.42 \times 10^{-10}$ & $2.69 \times 10^{-8}$ & $2.35 \times 10^{-7}$ & $3.35 \times 10^{-6}$ & $1.21 \times 10^{-5}$ \\
0.2 & $3.48 \times 10^{-10}$ & $1.61 \times 10^{-8}$ & $1.30 \times 10^{-7}$ & $1.85 \times 10^{-6}$ & $6.68 \times 10^{-6}$ \\
0.3 & $6.14 \times 10^{-11}$ & $2.81 \times 10^{-10}$ & $-2.38 \times 10^{-8}$ & $-3.40 \times 10^{-7}$ & $-1.23 \times 10^{-6}$ \\
0.4 & $-2.65 \times 10^{-10}$ & $-1.73 \times 10^{-8}$ & $-1.91 \times 10^{-7}$ & $-2.72 \times 10^{-6}$ & $-9.86 \times 10^{-6}$ \\
0.5 & $-5.55 \times 10^{-10}$ & $-3.20 \times 10^{-8}$ & $-3.24 \times 10^{-7}$ & $-4.61 \times 10^{-6}$ & $-1.67 \times 10^{-5}$ \\
0.6 & $-7.13 \times 10^{-10}$ & $-3.81 \times 10^{-8}$ & $-3.61 \times 10^{-7}$ & $-5.13 \times 10^{-6}$ & $-1.86 \times 10^{-5}$ \\
0.7 & $-6.19 \times 10^{-10}$ & $-2.81 \times 10^{-8}$ & $-2.25 \times 10^{-7}$ & $-3.20 \times 10^{-6}$ & $-1.62 \times 10^{-5}$ \\
0.8 & $-1.35 \times 10^{-10}$ & $6.39 \times 10^{-9}$ & $1.79 \times 10^{-7}$ & $2.46 \times 10^{-6}$ & $8.97 \times 10^{-6}$ \\
0.9 & $9.03 \times 10^{-10}$ & $7.55 \times 10^{-8}$ & $9.44 \times 10^{-7}$ & $1.34 \times 10^{-6}$ & $4.86 \times 10^{-5}$ \\
1.0 & $2.68 \times 10^{-9}$ & $1.90 \times 10^{-7}$ & $2.20 \times 10^{-6}$ & $3.12 \times 10^{-5}$ & $1.14 \times 10^{-4}$ \\
\hline \hline
\end{tabular}

TABle 5. Comparison of the Residual of FTD when $\beta=0.2$

\begin{tabular}{llcllc}
\hline \hline$x$ & $\psi=0.1$ & $\psi=0.2$ & $\psi=0.3$ & $\psi=0.4$ & $\psi=0.5$ \\
\hline 0.0 & $-1.71 \times 10^{-9}$ & $7.16 \times 10^{-8}$ & $7.18 \times 10^{-7}$ & $1.36 \times 10^{-6}$ & $4.36 \times 10^{-6}$ \\
0.1 & $-1.72 \times 10^{-9}$ & $6.23 \times 10^{-8}$ & $6.18 \times 10^{-7}$ & $1.17 \times 10^{-6}$ & $3.77 \times 10^{-6}$ \\
0.2 & $-1.71 \times 10^{-9}$ & $3.62 \times 10^{-8}$ & $3.39 \times 10^{-7}$ & $6.48 \times 10^{-7}$ & $2.09 \times 10^{-6}$ \\
0.3 & $-1.60 \times 10^{-9}$ & $-2.03 \times 10^{-9}$ & $-6.42 \times 10^{-8}$ & $-1.13 \times 10^{-7}$ & $-3.49 \times 10^{-7}$ \\
0.4 & $1.27 \times 10^{-9}$ & $-4.41 \times 10^{-8}$ & $-5.04 \times 10^{-7}$ & $-9.45 \times 10^{-7}$ & $-5.19 \times 10^{-6}$ \\
0.5 & $-5.41 \times 10^{-10}$ & $-7.86 \times 10^{-8}$ & $-8.52 \times 10^{-7}$ & $-1.61 \times 10^{-6}$ & $-3.03 \times 10^{-6}$ \\
0.6 & $8.30 \times 10^{-10}$ & $-9.08 \times 10^{-8}$ & $9.47 \times 10^{-7}$ & $-1.80 \times 10^{-6}$ & $-5.85 \times 10^{-6}$ \\
0.7 & $3.12 \times 10^{-9}$ & $-6.29 \times 10^{-8}$ & $-5.88 \times 10^{-7}$ & $-1.14 \times 10^{-6}$ & $-3.73 \times 10^{-6}$ \\
0.8 & $6.68 \times 10^{-9}$ & $2.66 \times 10^{-8}$ & $4.61 \times 10^{-7}$ & $8.49 \times 10^{-7}$ & $2.72 \times 10^{-6}$ \\
0.9 & $1.19 \times 10^{-8}$ & $2.02 \times 10^{-7}$ & $2.47 \times 10^{-6}$ & $4.71 \times 10^{-6}$ & $1.53 \times 10^{-5}$ \\
1.0 & $1.92 \times 10^{-8}$ & $4.92 \times 10^{-7}$ & $5.76 \times 10^{-6}$ & $1.08 \times 10^{-5}$ & $3.64 \times 10^{-5}$ \\
\hline \hline
\end{tabular}

TABLE 6 . Comparison of the Residual of FTD when $\beta=0.3$

\begin{tabular}{llcccc}
\hline \hline$x$ & $\psi=0.1$ & $\psi=0.2$ & $\psi=0.3$ & $\psi=0.4$ & $\psi=0.5$ \\
\hline 0.0 & $-3.46 \times 10^{-9}$ & $6.75 \times 10^{-8}$ & $7.17 \times 10^{-7}$ & $3.59 \times 10^{-6}$ & $1.35 \times 10^{-5}$ \\
0.1 & $-3.36 \times 10^{-9}$ & $5.91 \times 10^{-8}$ & $6.44 \times 10^{-7}$ & $3.10 \times 10^{-6}$ & $1.66 \times 10^{-5}$ \\
0.2 & $-3.06 \times 10^{-9}$ & $3.55 \times 10^{-8}$ & $5.55 \times 10^{-7}$ & $6.48 \times 10^{-7}$ & $2.09 \times 10^{-6}$ \\
0.3 & $-2.47 \times 10^{-9}$ & $8.09 \times 10^{-10}$ & $3.05 \times 10^{-8}$ & $1.71 \times 10^{-6}$ & $6.42 \times 10^{-6}$ \\
0.4 & $-1.49 \times 10^{-9}$ & $-3.77 \times 10^{-8}$ & $-5.79 \times 10^{-8}$ & $-3.19 \times 10^{-7}$ & $-1.20 \times 10^{-6}$ \\
0.5 & $2.68 \times 10^{-11}$ & $-7.01 \times 10^{-8}$ & $-7.65 \times 10^{-7}$ & $-2.52 \times 10^{-6}$ & $-9.49 \times 10^{-6}$ \\
0.6 & $2.29 \times 10^{-9}$ & $-8.35 \times 10^{-8}$ & $-8.49 \times 10^{-7}$ & $-4.27 \times 10^{-6}$ & $-1.61 \times 10^{-5}$ \\
0.7 & $5.57 \times 10^{-9}$ & $-6.20 \times 10^{-8}$ & $-5.27 \times 10^{-7}$ & $-4.75 \times 10^{-6}$ & $-1.78 \times 10^{-5}$ \\
0.8 & $1.01 \times 10^{-8}$ & $1.28 \times 10^{-8}$ & $4.14 \times 10^{-7}$ & $2.29 \times 10^{-6}$ & $8.66 \times 10^{-6}$ \\
0.9 & $1.63 \times 10^{-8}$ & $1.63 \times 10^{-7}$ & $2.22 \times 10^{-6}$ & $1.24 \times 10^{-5}$ & $4.67 \times 10^{-5}$ \\
1.0 & $2.45 \times 10^{-8}$ & $4.12 \times 10^{-7}$ & $5.17 \times 10^{-6}$ & $2.88 \times 10^{-5}$ & $1.09 \times 10^{-4}$ \\
\hline \hline
\end{tabular}

TABLE 7. Comparison of the Residual of FTD when $\beta=0.4$

\begin{tabular}{lccccc}
\hline \hline$x$ & $\psi=0.1$ & $\psi=0.2$ & $\psi=0.3$ & $\psi=0.4$ & $\psi=0.5$ \\
\hline 0.0 & $-2.49 \times 10^{-9}$ & $4.51 \times 10^{-8}$ & $3.72 \times 10^{-7}$ & $2.20 \times 10^{-6}$ & $8.74 \times 10^{-6}$ \\
0.1 & $-2.41 \times 10^{-9}$ & $4.01 \times 10^{-8}$ & $3.21 \times 10^{-7}$ & $1.89 \times 10^{-6}$ & $7.53 \times 10^{-6}$ \\
0.2 & $-2.15 \times 10^{-9}$ & $2.58 \times 10^{-8}$ & $1.76 \times 10^{-7}$ & $4.66 \times 10^{-6}$ & $4.14 \times 10^{-6}$ \\
0.3 & $-1.68 \times 10^{-9}$ & $4.66 \times 10^{-9}$ & $-3.37 \times 10^{-8}$ & $-1.90 \times 10^{-7}$ & $-7.87 \times 10^{-7}$ \\
0.4 & $-9.31 \times 10^{-10}$ & $-1.94 \times 10^{-8}$ & $-2.62 \times 10^{-7}$ & $-1.54 \times 10^{-6}$ & $-6.14 \times 10^{-6}$ \\
0.5 & $1.73 \times 10^{-10}$ & $-4.07 \times 10^{-8}$ & $-4.42 \times 10^{-7}$ & $-2.60 \times 10^{-6}$ & $-1.04 \times 10^{-5}$ \\
0.6 & $1.74 \times 10^{-9}$ & $-5.23 \times 10^{-8}$ & $-4.91 \times 10^{-7}$ & $-2.89 \times 10^{-6}$ & $-1.15 \times 10^{-5}$ \\
0.7 & $3.92 \times 10^{-9}$ & $-4.54 \times 10^{-8}$ & $-3.04 \times 10^{-7}$ & $-1.81 \times 10^{-6}$ & $-7.16 \times 10^{-6}$ \\
0.8 & $6.85 \times 10^{-8}$ & $-9.63 \times 10^{-9}$ & $2.40 \times 10^{-7}$ & $1.38 \times 10^{-6}$ & $5.62 \times 10^{-6}$ \\
0.9 & $1.07 \times 10^{-8}$ & $6.69 \times 10^{-8}$ & $1.28 \times 10^{-6}$ & $7.50 \times 10^{-6}$ & $1.47 \times 10^{-5}$ \\
1.0 & $1.58 \times 10^{-8}$ & $1.98 \times 10^{-7}$ & $2.98 \times 10^{-6}$ & $1.75 \times 10^{-5}$ & $7.02 \times 10^{-5}$ \\
\hline \hline
\end{tabular}


TABLE 8. Comparison of the Residual of FTD when $\beta=0.5$

\begin{tabular}{llllll}
\hline \hline$x$ & $\psi=0.1$ & $\psi=0.2$ & $\psi=0.3$ & $\psi=0.4$ & $\psi=0.5$ \\
\hline 0.0 & $-1.06 \times 10^{-7}$ & $-5.11 \times 10^{-8}$ & $4.83 \times 10^{-8}$ & $4.50 \times 10^{-7}$ & $2.55 \times 10^{-6}$ \\
0.1 & $-1.01 \times 10^{-7}$ & $-4.87 \times 10^{-8}$ & $4.16 \times 10^{-8}$ & $3.86 \times 10^{-7}$ & $2.19 \times 10^{-6}$ \\
0.2 & $-8.44 \times 10^{-8}$ & $-4.15 \times 10^{-8}$ & $2.28 \times 10^{-8}$ & $2.09 \times 10^{-7}$ & $1.19 \times 10^{-6}$ \\
0.3 & $-5.81 \times 10^{-8}$ & $-2.94 \times 10^{-8}$ & $-4.50 \times 10^{-9}$ & $-4.92 \times 10^{-8}$ & $-2.66 \times 10^{-7}$ \\
0.4 & $-2.12 \times 10^{-8}$ & $-1.21 \times 10^{-8}$ & $-3.41 \times 10^{-8}$ & $-3.28 \times 10^{-7}$ & $-1.84 \times 10^{-6}$ \\
0.5 & $2.63 \times 10^{-8}$ & $1.08 \times 10^{-8}$ & $-5.76 \times 10^{-8}$ & $-5.47 \times 10^{-7}$ & $-3.08 \times 10^{-6}$ \\
0.6 & $8.44 \times 10^{-8}$ & $3.97 \times 10^{-8}$ & $-6.38 \times 10^{-8}$ & $-6.00 \times 10^{-7}$ & $-3.39 \times 10^{-6}$ \\
0.7 & $1.53 \times 10^{-7}$ & $7.53 \times 10^{-8}$ & $-3.94 \times 10^{-8}$ & $-3.59 \times 10^{-7}$ & $-2.05 \times 10^{-6}$ \\
0.8 & $2.32 \times 10^{-7}$ & $1.18 \times 10^{-7}$ & $3.16 \times 10^{-8}$ & $3.27 \times 10^{-7}$ & $1.80 \times 10^{-6}$ \\
0.9 & $3.22 \times 10^{-7}$ & $1.69 \times 10^{-7}$ & $1.68 \times 10^{-7}$ & $1.63 \times 10^{-6}$ & $1.44 \times 10^{-6}$ \\
1.0 & $4.23 \times 10^{-7}$ & $2.29 \times 10^{-7}$ & $3.89 \times 10^{-7}$ & $3.76 \times 10^{-6}$ & $2.11 \times 10^{-5}$ \\
\hline \hline
\end{tabular}

\section{References}

[1] S. Abbasbandy, E. Shivanian and I. Hashim, Exact analytical solution of forced convection in a porous-saturated duct, Commun. Nonlinear Sci. Numer. Simul. 16 (2011), 3981-3989.

[2] L. Ali, S. Islam, T. Gul, I. Khan and L.C.C. Dennis, New version of Optimal Homotopy Asymptotic Method for the solution of nonlinear boundary value problems in finite and infinite intervals, Alexandria Eng. J. 55 (2016), 2811-2819.

[3] J. Ali, S. Islam, S. Islam and G. Zamand, The solution of multipoint boundary value problems by the Optimal Homotopy Asymptotic Method, Comput. Math. Appl. 59 (2010), 2000-2006.

[4] C. Arslanturk, A decomposition method for fin efficiency of convective straight fins with temperature-dependent thermal conductivity, Int. Commun. Heat Mass Transfer 32 (2005), 831-841.

[5] M.M. Awartani and M.H. Hamdan, Fully developed flow through a porous channel bounded by flat plates, Appl. Math. Comput. 169 (2005), 749-757.

[6] I. Aziz and Q. Ul Ain, Numerical solution of partial integro-differential equations with weakly singular kernels, Adv. Math. Models Appl. 5(2) (2020), 149-160.

[7] A. Aziz and S.M. Enamul Hug, Perturbation solution for convecting fin with variable thermal conductivity, J. Heat Transfer 97 (1975), 300-311.

[8] R. Baby and C. Balaji, Experimental investigations on phase change material based finned heat sinks for electronic equipment cooling, Int. J. Heat Mass Transfer $\mathbf{5 5}$ (2012), 1642-1649.

[9] M.N. Bouaziz, S. Recnak, S. Hanini, Y. Bal and K. Bal, Numerical study of nonlinear heat transfer in longitudinal fins, Int. J. Thermal Sci. 40 (2001), 843-857.

[10] V.V. Calmidi and R.L. Mahajan, Forced convection in high porosity metal foams, ASME J. Heat Transfer 122 (2000), 557-565.

[11] S.B. Coşkun and M.T. Atay, Fin efficiency analysis of convective straight fins with temperature dependent thermal conductivity using variational iteration method, Appl. Therm. Eng. 28 (2008), 2345-2352.

[12] M. Dehghan and J. Manafian, The solution of the variable coefficients fourth-order parabolic partial differential equations by homotopy perturbation method, Z. Naturforsch. 64 (2009), 420-430.

[13] M. Dehghan and J. Manafian Heris, Study of the wave-breaking's qualitative behavior of the Fornberg-Whitham equation via quasi-numeric approaches, Int J. Num. Methods Heat Fluid Flow 21 (2011), 736-753.

[14] M. Dehghan, J. Manafian and A. Saadatmandi, Solving nonlinear fractional partial differential equations using the homotopy analysis method, Num. Meth. Partial Dif. Eq J. 26 (2010), 448-479. 
[15] M. Dehghan, J. Manafian and A.Saadatmandi, The solution of the linear fractional partial differential equations using the homotopy analysis method, Z. Naturforsch. 65a (2010), 935-949.

[16] M. Dehghan, J. Manafian and A. Saadatmandi, Application of semi-analytic methods for the Fitzhugh-Nagumo equation, which models the transmission of nerve impulses, Math. Meth. Appl. Sci. 33 (2010), 1384-1398.

[17] M. Dehghan, J. Manafian and A. Saadatmandi, Application of semi-analytical methods for solving the Rosenau-Hyman equation arising in the pattern formation in liquid drops, Int. J. Num. Methods Heat Fluid Flow 22 (2012), 777-790.

[18] M. Dehghan, J. Manafian and A. Saadatmandi, Application of semi-analytical methods for solving the Rosenau-Hyman equation arising in the pattern formation in liquid drops, Int. J. Num. Methods Heat Fluid Flow 22 (2012), 537-553.

[19] M. Esmaeilpour and D.D. Ganji, Solution of the Jeffery-Hamel flow problem by optimal homotopy asymptotic method, Comput. Math. Appl. 59 (2010), 3405-3411.

[20] A.D.D. Ganji, B.G.A. Afrouzi and R. Talarposhti, Application of variational iteration method and homotopy-perturbation method for nonlinear heat diffusion and heat transfer equations, Phys. Lett. A 368 (2007), 450-457.

[21] R.M. Ganji and H. Jafari, Numerical solution of variable order integro-differential equations, Adv. Math. Models Appl. 4(1) (2019), 64-69.

[22] H.F. Guliyev and Z.R. Safarova, On a determination of the initial functions from the observed values of the boundary functions for the second-order hyperbolic equation, Adv. Math. Models Appl. 3(3) (2018), 215-222.

[23] K. Günel and İ. Gör, Gravitational search algorithm solutions of initial value problems for ordinary differential equations, Adv. Math. Models Appl. 4(3) (2019), 232242.

[24] M.S. Hashmi, N. Khan and S. Iqbal, Optimal homotopy asymptotic method for solving nonlinear Fredholm integral equations of second kind, Appl. Math. Comput. 218 (2012), 10982-10989.

[25] J.H. He, Variational approach to the sixth-order boundary value problems, Appl. Math. Comput. 143 (2003), 537-538.

[26] J.H. He, A coupling method for homotopy technique and perturbation technique for nonlinear problem, Int. J. Nonlinear Mech. 35 (2000), 37-43.

[27] J.H. He, Asymptotology by homotopy perturbation method, Appl. Math. Comput. 156 (2004), 591-596.

[28] J.H. He, Homotopy perturbation method for solving boundary problems, Phys. Lett. A 350 (2006), 87-88.

[29] N. Herisanu and V. Marinca, Accurate analytical solutions to oscillators with discontinuities and fractional-power restoring force by means of the optimal homotopy asymptotic method, Comput. Math. Appl. 60 (2010), 1607-1615.

[30] N. Herisanu and V. Marinca, Explicit analytical approximation to large-amplitude non-linear oscillations of a uniform cantilever beam carrying an intermediate lumped mass and rotary inertia, Meccanica 45 (2010), 847-855.

[31] N. Herisanu, V. Marinca, T. Dordea and G. Madescu, A new analytical approach to nonlinear vibration of an electric machine, Proc. Romanian. Acad. Ser. A: Math. Phys. Tech. Sci., Inf. Sci. 9 (3) (2008), 229-236.

[32] K. Hooman, A perturbation solution for forced convection in a porous-saturated duct, J. Comp. Appl. Math. 211 (2008), 57-66.

[33] K. Hooman and A.A. Ranjbar-Kani, A perturbation based analysis to investigate forced convection in a porous saturated tube, J. Comput. Appl. Math. 162 (2004), 411-479. 
[34] K. Hooman, J. Li and M. Dahari, Thermal dispersion effects on forced convection in a porous-saturated pipe, Thermal Sci. Eng. Prog. 2 (2017) 64-67.

[35] M. Idrees, S. Islam, S.I.A. Tirmizi and Sirajul Haqa, Application of the optimal homotopy asymptotic method for the solution of the Korteweg-de Vries equation, Math. Comput. Model. 55 (2012), 1324-1333.

[36] M. Inc, Application of homotopy analysis method for fin efficiency of convective straight fins with temperature-dependent thermal conductivity, Math. Comput. Simul. 79 (2008), 189-200.

[37] M. Kaviany, Laminar flow through a porous channel bounded by isothermal parallel plates, Int. J. Heat Mass Transfer 28 (1985), 851-858.

[38] F. Khani, M.A. Raji and S. Hamedi-Nezhad, A series solution of the fin problem with a temperature-dependent thermal conductivity, Commun. Nonlinear Sci. Numer. Simul. 14 (2009), 3007-3017.

[39] S. Kim and C.H. Huang, A series solution of the fin problem with a temperaturedependent thermal conductivity, J. Phys. D: Appl. Phys. 39 (2006), 4894-4901.

[40] S.J. Liao, The proposed homotopy analysis technique for the solution of nonlinear problems, Ph.D. Thesis, Shanghai Jiao Tong University (1992).

[41] E. Magyari, B. Keller and I. Pop, Exact analytical solutions of forced convection flow in a porous medium, Int. Commun. Heat Mass Transfer 28 (2001), 233-241.

[42] J. Manafian, Solving the integro-differential equations using the modified Laplace Adomian decomposition method, J. Math. Ext. 6 (2012), 1-15.

[43] J. Manafian and S. Heidari, Periodic and singular kink solutions of the Hamiltonian amplitude equation, Adv. Math. Models Appl. 4(2) (2019), 134-149.

[44] V. Marinca, N. Herisanu, C. Bota and B. Marinca, An optimal homotopy asymptotic method applied to the steady flow of fourth-grade fluid past a porous plate, Appl. Math. Lett. 22 (2) (2009), 245-251.

[45] V. Marinca, N. Herisanu and I. Nemes, Optimal homotopy asymptotic method with application to thin film flow, Cent. Eur. J. Phys. 6 (3) (2008), 648-653.

[46] M. S. Mechee and K. B. Mussa, Generalization of rkm integrators for solving a class of eighth-order ordinary differential equations with applications, Adv. Math. Models Appl. 5(1) (2020), 111-120.

[47] M. Moosavi, M. Momeni, T. Tavangar, R. Mohammadyari and M. Rahimi-Esbo, Variational iteration method for flow of non-newtonian fluid on a moving belt and in a collector, Alexandria Eng. J. 55 (2016), 1775-1783.

[48] S.S. Motsa, P. Sibanda and S. Shateyi, A new spectral-homotopy analysis method for solving a nonlinear second order BVP, Commun. Nonlinear Sci. Num. Simul. 15 (2010), 2293-2302.

[49] A.H. Nayfeh, Problems in Perturbation, second ed., Wiley, NewYork, 1993.

[50] D.A. Nield, The boundary condition for the Rayleigh-Darcy problem: limitation of the Brinkman equation, J. Fluid Mech. 128 (1983), 37-40.

[51] D.A. Nield and A. Bejan, Convection in Porous Media, third ed., Springer, NewYork, 2006.

[52] D.A. Nield, S.L.M. Junqueira and J.L. Lage, Forced convection in a fluid-saturated porous-medium channel with isothermal or isoflux boundaries, J. Fluid Mech. 322 (1996), 201-214.

[53] B. Parsa, M.M. Rashidi, O. Anwar Bég and S.M. Sadri, Semi-computational simulation of magneto-Hemodynamic flow in a semi-porous channel using optimal homotopy and differential transform methods, Comput. Bio. Medicine 43 (2013), 11421153 . 
[54] A. Rajabi, Homotopy perturbation method for fin efficiency of convective straight fins with temperature-dependent thermal conductivity, Phys. Lett. A 364 (2007), 33-37.

[55] P. Razelos and K. Imre, The optimum dimensions of circular fins with variable thermal parameters, J. Heat Transfer 102 (1980), 420-425.

[56] M. Shaban, S. Kazem and J.A. Rad, A modification of the homotopy analysis method based on Chebyshev operational matrices, Math. Comput. Model. 57 (2013), 1227-1239.

[57] R.K. Shah and A.L. London, Laminar Flow Forced Convection in Ducts (Advances in Heat Transfer, Supplement 1), Academic Press, NewYork, 1978.

[58] S. Sohrabpour and A. Razani, Optimization of convective fin with temperaturedependent thermal parameters, J. Franklin Institute 330 (1993), 37-49.

[59] M. Tatari and M. Dehgan, The use of the Adomian decomposition method for solving multipoint boundary value problems, Phys. Scr. 73 (2006), 672-676.

[60] H. Temimi and A. Ansari, A semi analytical iterative technique for solving nonlinear problems, J. Comput. Math. Appl. 61 (2011), 203-210.

[61] K. Vafai and S.J. Kim, Forced convection in a channel filled with porous medium: an exact solution, ASME J. Heat Transfer 111 (1989), 1103-1106.

[62] K. Vafai and S.J. Kim, Thermal dispersion effects on forced convection in a poroussaturated pipe, Thermal Sci. Eng. Prog. 2 (2017), 64-70.

[63] S. Sh. Yusubov, Boundary value problems for hyperbolic equations with a caputo fractional derivative, Adv. Math. Models Appl. 5(2) (2020), 192-204.

\section{Jalil Manafian}

Department of Applied Mathematics, Faculty of Mathematical Sciences, University of Tabriz, Tabriz, Iran

E-mail address: j_manafianheris@tabrizu.ac.ir

Received: November 16, 2020; Revised: February 4, 2021; Accepted: March 4, 2021 